\title{
Weshalb nach Julian die mosaisch-christliche Schöpfungslehre der platonischen Demiurgie unterlegen ist
}

\author{
Jan Opsomer ${ }^{1}$
}

1. Einleitung - Julian beweist: Jahwe, doch nicht der, der er ist...

In seinem leider nur indirekt und fragmentarisch überlieferten Werk Gegen die Galiläer unterzieht Julian die mosaische Schöpfungsdarstellung einem systematischen Vergleich mit der angeblich wissenschaftlich wie theologisch weit überlegenen platonischen Weltentstehungslehre. ${ }^{2}$ Julians Ziel ist es zu zeigen, dass die alttestamentarische Schöpfungsgeschichte in wesentlichen Hinsichten hinter den Standards einer präzisen philosophischen Darstellung zurückbleibt. So habe der jüdische Schöpfergott alle Merkmale einer lokalen demiurgischen Gottheit, die jedoch von Moses zu Unrecht für den höchsten und sogar für den einzigen Gott gehalten wird. Damit bringe der Verfasser des Buches Genesis lediglich seine Unkenntnis des wahren Baumeisters, dessen Werke nur in Platons Timaios auf angemessene Weise Berücksichtigung finden, zum Ausdruck. Aber auch wenn der in Genesis dargebotene Gott der universelle Weltschöpfer gewesen wäre, so dürfe er nicht mit dem höchsten Gott gleichgesetzt werden, denn, wie wir nachher darlegen werden, ist auch der platonische Demiurg - Julian und seinen platonischen Lehrern zufolge - nicht mit dem höchsten göttlichen Prinzip identisch. Auch hinsichtlich dieser Einsicht bleibe somit die jüdische hinter der platonischen Theologie zurück.

Julians Invektive beschränkt sich in diesem Punkt allerdings nicht auf eine bloße Ablehnung des Monotheismus zugunsten eines polytheistischen Gottesverständ-

1 Ich möchte ganz herzlich Rainer Schäfer für die sprachliche Durchsicht meines Textes sowie Gabor Buzasi für zahlreiche Anregungen danken.

2 Siehe Contra Galileos, Fragment 6,3-10 [49A]. Diese Schrift wird nach der Edition von Masaracchia zitiert (Giuliano Imperatore, Contra Galilaeos, Hg. E. Masaracchia (Testi e commenti, 9), Roma 1990). Die anderen Schriften werden nach der Edition in der Budé-Reihe zitiert: L'Empereur Julien. Cuvres complètes, Tome II - 1re partie, Discours de Julien Empereur. À Thémistius - Contre Héracleios le Cynique - Sur la Mère des Dieux - Contre les Cyniques ignorants, Hg. G. Rochefort; 2e partie, Discours de Julien Empereur. Les Césars - Sur HéliosRoi - Le Misopogon, Hg. Chr. Lacombrade (Collection des Universités de France), Paris 19631964. 
nisses. Er sucht ebenfalls anhand einer vergleichenden Exegese nachzuweisen, dass sich die Macht und Wirkung des biblischen Schöpfers nur auf ein beschränktes Teilgebiet der dem platonischen Demiurgen zukommenden Zuständigkeiten erstrecken. Julian räumt durchaus ein, dass auch die alttestamentarischen, später von den Christen übernommenen Gottesvorstellungen, insofern sie nämlich mit den altüberlieferten griechischen theologischen Vorstellungen verträglich sind, einen Wahrheitskern enthalten. ${ }^{3}$ In dieser vergleichenden Betrachtung stellt sich aber sofort heraus, dass es sich in Genesis eigentlich nur um den ,angrenzenden“, unmittelbaren Demiurgen dieser Welt handeln kann (

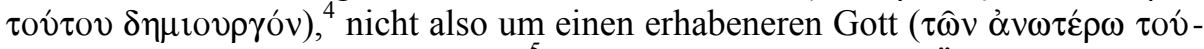

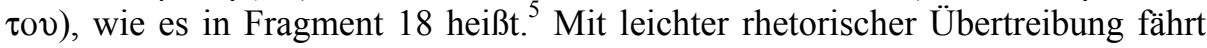
der Verfasser fort, Moses habe sich nicht getraut, etwas über die Natur der Engel Hinausgehendes zu behaupten. Nicht einmal zu den Fragen, ob sie entstanden oder vielmehr unentstanden sind, und ob sie der selben Gottheit dienen, die sie auch hervorgebracht hat, habe Moses eindeutig Stellung bezogen. ${ }^{6}$ Aber nicht nur hinsichtlich dieser Probleme bleibe der Bericht des Moses unbefriedigend, sondern vor allem mit Bezug auf die Art und Weise, in welcher der Gott die in den ersten Versen des Buchs Genesis genannten Entitäten ${ }^{7}$ angeblich hervorgebracht hat, lässt die Beschreibung noch viel zu wünschen übrig. Bestimmte Dinge, wie der Tag, die Sterne ${ }^{8}$ und das Firmament, entstehen anscheinend auf einfachen

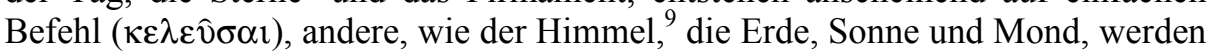
hervorgebracht $(\pi \mathrm{ol} \hat{\eta} \sigma \alpha)$, wiederum andere waren bereits da, aber blieben ver-

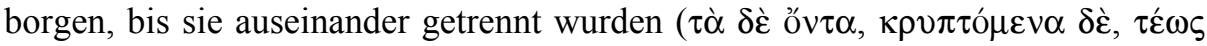
$\delta 1 \alpha \kappa \rho \hat{\imath} v \alpha \imath$ ), wie es der Fall zu sein scheint für das Wasser und das Trockene. Zur Entstehungsweise des über dem Wasser schwebenden Geistes $(\pi \nu \varepsilon \hat{v} \mu \alpha)$ hingegen habe Moses es gar nicht gewagt sich zu äußern. Es sei nicht einmal klar, ob er dann überhaupt entstanden sei oder nicht. ${ }^{10}$

3 Siehe Contra Galileos, Fragment 18,2-4 [96C].

4 Contra Galileos, Fragment 18,4-5 [96C]. Wegen der schlechten Überlieferungslage ist der Gedankengang hier nicht ganz klar. Aus dem Kontext ergibt sich jedoch, dass dem Verfasser zufolge der alttestamentarische Schöpfergott nur dieser angrenzende Demiurg und nicht der höchste Gott sein kann.

5 Contra Galileos, Fragment 18,5 [96C]. Ich bevorzuge die Lesart der Manuskripte statt Masarac-

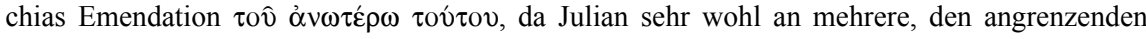
Demiurgen übersteigende Götter denken könnte, auch wenn Cyrillus, unsere Quelle, einige Zeilen vorher Julians an Moses gerichteten Vorwurf mit einem Verweis auf den höchsten Gott be-

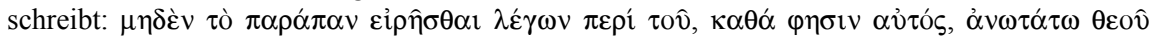
(Contra Iulianum 96A).

6 Vergleiche aber auch Contra Galileos, Fragment 67 [290B-291B]. Siehe auch Anmerkung 40.

7 Julian zitiert Genesis 1,1-18 in Fragment 6,11-25 [49B-D].

8 Siehe Masaracchia (Anmerkung 2), S. 54-55.

9 Mit ,Himmel' bezeichne ich hier und im Folgenden den oberen Teil des sichtbaren Universums (oủpavós), das heißt den supralunaren Bereich.

10 Siehe Contra Galileos, Fragment 18,5-18 [96C-E]. Vergleiche Celsus apud Origenem, contra Celsum. 6,53. 
Dieses Fragment der julianischen Streitschrift dokumentiert somit genau, in welchen Erwartungen ein philosophisch gebildeter Leser sich Julian zufolge bei der Lektüre des biblischen Schöpfungsberichtes enttäuscht sehen wird: Ein Schöpfungsbericht sollte entstandene und unentstandene Entitäten, wenn es denn solche gibt, eindeutig voneinander unterscheiden und die demiurgischen Tätigkeiten dementsprechend präzise benennen. Es macht dabei einen wesentlichen Unterschied, ob der Demiurg etwas hervorbringt oder Vorhandenes umdisponiert und ordnet. Auch eine andere Angriffslinie ist bereits angedeutet: Wenn der Demiurg mit Vorhandenem arbeiten muss und es unentstandene, das heißt gleichewige Entitäten gibt, könnte er wohl kaum der höchste Gott sein.

Diese Einwände werden in Fragment 6 [48E-49E] bestätigt. Moses, der angibt, von Angesicht zu Angesicht mit Gott selbst geredet zu haben, ${ }^{11}$ setzt einfach am Anfang seines Schöpfungsberichts die Existenz bestimmter Dinge voraus. Wer aber das Entstehen des Lichtes erwähnt, sollte auch die Existenz der Finsternis, der Tiefe und des Wassers erklären. Das Fehlen einer Stellungnahme zur Frage der Seinsart beziehungsweise des Entstehens der Engel führt den Kaiser dazu, seine Kritik an Moses noch zu verschärfen: Diese Lücke sei nicht dem Zufall zu überlassen, sondern demonstriere, dass Moses seinen Demiurgen wesentlich als den Gestalter körperlicher Dinge betrachtet. Er bringe keine unkörperlichen Wesen, wie die Engel, hervor, sondern lediglich die Himmelskörper sowie die irdischen Dinge. Überdies arbeite er mit vorhandener Materie, die er gestaltet. $^{12}$

Als eine der bedeutendsten Timaios-Stellen hebt Julian die Rede des Demiurgen an die so genannten Jungen Götter hervor (Timaios 41A7-D3), in der er ihnen den Auftrag erteilt, seine Arbeit in denjenigen Bereichen weiterzuführen, die nicht an seiner Unsterblichkeit teilhaben sollten. Dies gilt Timaios zufolge insbesondere für den Menschen, der nur in seinem höchsten, führenden und göttlich genannten Seelenteil eine $\mathrm{Art}^{13}$ Unsterblichkeit genießt. Die von Timaios dargebotene Rede des Demiurgen bezeuge, so der Kaiser, die Differenziertheit, die die platonische Behandlung des Themas auszeichnet. In der genannten Passage erwähnt der Demiurg sichtbare Götter, mit denen er die Sonne, den Mond, die Sterne und das Himmelsgewölbe bezeichne. Diese seien aber lediglich Abbilder der unsichtbaren Götter. So entspreche der sichtbaren eine unsichtbare, geistige Son-

11 Siehe Contra Galileos, Fragment 6,9-10 [49A-B].

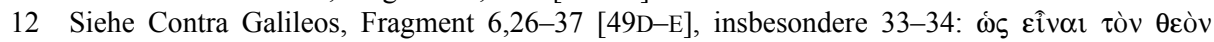

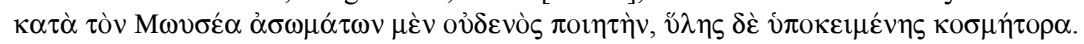

13 Spätere Platoniker haben Platons Verwendung des Ausdruckes $\kappa \alpha \theta^{\prime}$ ő $\sigma o v \mu \dot{\varepsilon} v \quad \alpha \jmath ̉ \tau \hat{\omega} v$

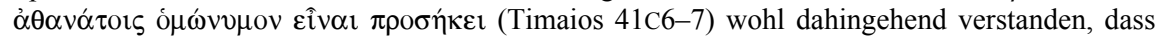
die Menschen nicht die gleiche, sondern eine abgeschwächte (das heißt eine von dem Willen des Demiurgen abhängig gemachte) Art der Unsterblichkeit besitzen, was mit der durch die aristotelische Kategorienschrift veranlassten Bedeutungsverengung des Adjektivs ó $\mu \omega ́ v \vartheta \mu \varsigma_{\text {zusam- }}$ menhängt. Die spätneuplatonische Deutung der genannten Timaiosstelle wird diskutiert in Opsomer, Jan, La démiurgie des jeunes dieux selon Proclus, in: Les Études Classiques, 71 (2003), S. 5-49, hier 27-35. 
ne und das Gleiche gelte für die anderen sichtbaren Götter. ${ }^{14}$ Somit lasse Platon ein erstaunliches Wissen über die Seins- und Entstehensweise der unsichtbaren Götter erkennen. Im Gegensatz zu Moses verstehe er, dass die unsichtbaren Götter in und mit dem Demiurgen sind, von ihm hervorgebracht werden und aus ihm hervorgehen. ${ }^{15}$ Den Beweis für diese These entdeckt Julian in der Anrede an die Jungen Götter beziehungsweise in der berühmten Formel ,Götter der Götter

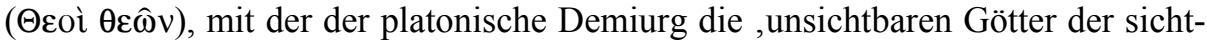
baren Götter ' bezeichne. Der platonische Demiurg ist der Schöpfer beider Götterklassen. Er verfertigt nicht nur den sichtbaren Himmel, die Erde, das Meer und die Sterne, sondern bringt ebenfalls deren unsichtbare Archetypen hervor. ${ }^{16}$ Was vom ersten Demiurgen hervorgebracht wird, ist notwendigerweise unsterblich. Hierzu zählen die Welt als ganze, die Himmelskörper, aber auch die in der Welt befindlichen rationalen Seelen der Menschen. Die anderen Götter, die im Anschluss an die Schöpfung der unsterblichen Weltkomponente als Demiurgen des Sterblichen auftreten werden, übernehmen die demiurgische $\mathrm{Kraft}^{17}$ von ihrem Vater und bringen die sterblichen Lebewesen sowie die sterblichen Teile des Menschen - Körper und irrationale Seele - hervor. ${ }^{18}$ Platon markiere somit eine deutliche Abstufung zwischen dem Demiurgen des Ganzen, in dessen schöpferischer Tätigkeit er zusätzlich zwei wesentliche Momente unterscheide, und den untergeordneten Demiurgen, die seine Arbeit weiterführen und im Gegensatz zum Ersteren imstande seien, Sterbliches entstehen zu lassen. Julian betont, dass man diese Unterscheidungen machen muss, denn sonst könnte man die Vielfältigkeit der Welt - die Differenz zwischen ewiger Welt und sterblichem Menschen sowie die Kluft zwischen Mensch und vernunftlosem Tier nicht erklären. Bestünde nur ein einziger Demiurg, wie es Moses zu glauben scheint, so wäre alles in der Welt genau so unsterblich und göttlich wie die Welt selbst. In Wahrheit aber gebe es diese ontologische Differenz sowie einen weiten Zwischenraum zwischen den

14 Siehe Contra Galileos, Fragment 10,4-9 [65A-B]. Dies entspricht der in der Heliosrede vertretenen Sichtweise, wobei allerdings in der Heliosrede die sichtbaren Götter wohl eher als Aspekte der unsichtbaren Götter denn als eigenständige Gottheiten betrachtet werden können, siehe unten.

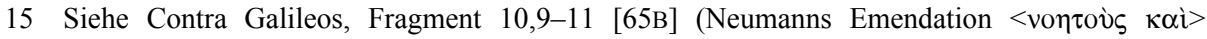

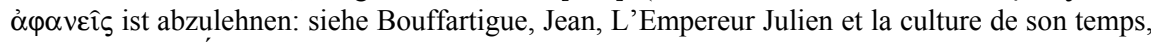
(Collection des Études Augustiniennes, Série Antiquité 133), Paris 1992, S. 396; es handelt sich hier nämlich nicht um noetische (intelligible) Götter im strengen Sinn, sondern um noerische (intellektive) Götter; siehe unten). Vergleiche Sallustius, De deis 13,4.

16 Siehe Contra Galileos, Fragment 10,11-15 [65B-C]. Masaracchias Ansicht ([Anmerkung 2], S. 51), der Demiurg könne nur die unsichtbare Welt hervorbringen, da diese die einzig wirklich seiende sei, während die Körper aus ihr durch Emanation hervorgehen, ist verfehlt.

17 Vergleiche Timaios 41C5.

18 Siehe Contra Galileos, Fragment 10,15-28 [65C-D]. Zur unterschiedlichen Herkunft der rationalen und der irrationalen Seele, vergleiche Iamblichus, De Mysteriis 8,6 (269,1-5); Sallustius, De deis 8,1; Iulianus, oratio 9 [6] (contra Cynicum) 3,9 [182D]; 13,36 [194D]; oratio 11 [4], 1,4 [130B]. 
Unsterblichen und den Sterblichen, ${ }^{19}$ das heißt eine vielstufige Hierarchie der göttlichen und sterblichen Lebewesen. ${ }^{20}$ Deswegen brauche man unterschiedliche effiziente Ursachen, das heißt mehrere Demiurgen. ${ }^{21}$

Die platonische Ansicht, dass die untergeordneten Demiurgen zuerst vom höheren Demiurgen hervorgebracht werden mussten, macht Julians bereits erwähnte Kritik, Moses habe keine Klarheit geschaffen hinsichtlich der Frage, ob die Engel entstanden sind, verständlicher. ${ }^{22}$ Im Gegensatz zu Moses habe Platon nicht nur klar gezeigt, dass die untergeordneten Götter ${ }^{23}$ entstanden sind, sondern auch, warum dies so sein musste. ${ }^{24}$ Sonst nämlich wären ihre Schöpfungen genauso unsterblich gewesen wie der Demiurg selber. Es war aber nötig, dass nicht nur Unsterbliches existiert. ${ }^{25}$

Das Lehrstück, die sichtbaren Götter beziehungsweise die Himmelskörper seien Abbilder noetischer Urbilder, ${ }^{26}$ wie auch die Vorstellung der unsichtbaren Götter beziehungsweise Weltursachen als in dem Demiurgen enthalten und aus ihm hervorgehend, ${ }^{27}$ findet man auch bei Jamblich, was, zusammen mit den zahl-

19 Siehe Contra Galileos, Fragment 10,28-34 [65E-66A].

20 Der durch den großen Abstand zwischen dem ersten Gott und unserer menschlichen Natur bedingte Bedarf an mittleren Instanzen wird ebenfalls durch Sallustios hervorgehoben: De deis 13,4-5. Dieser Gedanke geht auf Jamblich zurück: Siehe Iamblichus, In Timaeum, Fragment 60

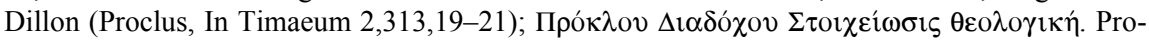
clus. The Elements of Theology, Hg. E.R. Dodds, Oxford 1963, S. xxii.

21 Siehe Contra Galileos, Fragment 10,33-34 [66A]. Vergleiche Smith, Rowland, Julian's gods. Religion and philosophy in the thought and action of Julian the Apostate, London/New York 1995, S. 195. Auch in oratio 8 [5], 3,12-13 [161D] betont Julian, dass es eine große Anzahl an Demiurgen beziehungsweise demiurgischen Kräften geben muss.

22 Hierzu siehe Smith (Anmerkung 21), S. 195.

23 Julian meint zeigen zu können, dass die mosaischen Engel als Götter betrachtet werden sollen. Siehe Contra Galileos, Fragment 67. Allerdings ist er ebenfalls der Meinung, dass Engel den regionalen Göttern zur Seite stehen. Siehe Anmerkung 40 und 152.

24 Julian ist vermutlich der Ansicht, dass die unsichtbaren, aber dennoch vom übergeordneten Demiurgen hervorgebrachten Götter, ihm nur ontologisch, nicht zeitlich nachgeordnet sind. Vergleiche Iamblichus, In Timaeum, Fragment 32 Dillon.

25 Vergleiche Timaios 41B7-C5. Möglicherweise argumentierte Julian im nicht erhaltenen Teil der Streitschrift, dass es für den höchsten Demiurgen wohl kaum passend ist, sich mit der Schöpfung der niedrigsten Geschöpfe beschäftigen zu müssen. Diese Kritik wird zum Beispiel auch von Kelsos geäußert, der zudem die mosaische Vorstellung rügt, Gott arbeite mit den Händen, werde müde und müsse sich von der schweren Arbeit erholen. Siehe Origenes, Contra Celsum 6,61; 64. Über die Ursprünge dieser Kritik innerhalb der platonischen Schule, siehe Opsomer, Jan, Demiurges in Early Imperial Platonism, in: Gott und die Götter bei Plutarch. Götterbilder Gottesbilder - Weltbilder, Hg. R. Hirsch-Luipold, Berlin/New York 2005, S. 51-99.

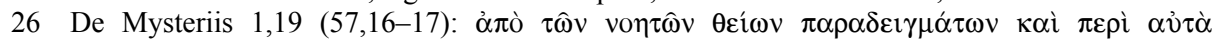

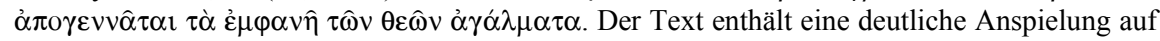
ps.-Platon, Epinomis 983E5-984A1.

27 Vergleiche Iamblichus, In Timaeum, Fragment 34 Dillon. Hierzu auch Opsomer, Jan, A Craftsman and his Handmaiden. Demiurgy According to Plotinus, in: Platons Timaios als Grundtext der Kosmologie in Spätantike, Mittelalter und Renaissance. Plato's Timaeus and the Foundations of Cosmology in Late Antiquity, the Middle Ages and Renaissance, Hg. Th. Leinkauf/C. 
reichen Parallelen zu Sallustios' Schrift Über die Götter und die Welt, ${ }^{28}$ den Gedanken nahe legt, die von Julian vertretene Demiurgieauffassung sei im Kern jamblicheisch. ${ }^{29}$ Wenn die Absicht der julianischen Streitschrift in der Verteidigung der tradierten Kultpraxen besteht, ${ }^{30}$ so geschieht dies zumindest teilweise auf der intellektuellen Basis des spätantiken Platonismus, wie er von Jamblich, genau auch zu diesem Zweck, weiterentwickelt worden war. ${ }^{31}$ Es handelt sich somit um eine philosophische Rechtfertigung der traditionellen Religion gegen die jüdischen und christlichen Exklusivitätsansprüche.

Nachdem, wie wir bereits gesehen haben, Julian argumentiert hat, die Darstellung der Schöpfungsgeschichte des Moses könne sich eigentlich lediglich auf den dem Kosmos unmittelbar angrenzenden Demiurgen beziehen (den unmittelbaren Demiurgen des Universums), und nicht, wie die Juden selbst meinen, auf den erhabeneren Schöpfergott, verschärft er jetzt seine Kritik, indem er nachzuweisen sucht, dass der jüdische Schriftsteller sich nicht einmal zu einer vollständigen Erörterung des ,angrenzenden ${ }^{632}$ Demiurgen und von dessen Werken imstande

Steel (Ancient and Medieval Philosophy. De Wulf Mansion Centre, Series 1 34), Leuven 2005, S. 67-102, hier 76-78.

28 Zur Autorschaft dieser Schrift, beziehungsweise zur Frage der Bekanntschaft Julians mit dem Autor, siehe Bouffartigue, Jean, Iulianus (Julien) l'Empereur, in: Dictionnaire des philosophes antiques, Hg. R. Goulet, III, d'Eccélos à Juvénal, Paris 2000, S. 961-978, hier 972, und die dort verzeichnete Literatur. Möglicherweise ist der Autor Sallustios identisch mit dem Adressat der Helios-Rede (erwähnt in 44,1 [157B], sowie im Titel der Schrift).

29 Vermutlich war Jamblichs Schrift Über die Götter Julians Hauptquelle für die platonische Demiurgielehre. Auch die Schrift Über die Rede des Zeus im Timaios kommt als Quelle in Betracht. Vergleiche Dillon, John M., Iamblichi Chalcidensis in Platonis dialogos commentariorum fragmenta (Philosophia antiqua 23), Leiden 1973, S. 23-24; Smith (Anmerkung 21), S. 144 und 265, Anmerkung 23. Siehe aber auch Asmus, Rudolf, Der Alkibiades-Kommentar des Jamblichos als Hauptquelle für Kaiser Julian (Sitzungsberichte der Heidelberger Akademie der Wissenschaften, Philosophisch-historische Klasse, Jahrgang 1917, 3. Abhandlung), Heidelberg 1917; Bregman, Jay, Elements of the Emperor Julian's Theology, in: Traditions of Platonism. Essays in Honour of John Dillon, Hg. J. J. Cleary, Aldershot 1999, S. 337-350, hier 343, Anmerkung 15.

30 Siehe Smith (Anmerkung 21), S. 205: „If my account holds good, the driving force behind Against the Galileans will be found in a keen defence of ancestral cultic practice focused upon sacrifice by one to whom it seemed, in all its diversity, the very cement of a civilized world." Allerdings ist zu bemerken, dass sich Julians Polemik hauptsächlich direkt auf Texte bezieht. Bei Kelsos hingegen stehen Doktrinen und Kultpraxen im Vordergrund. Siehe Bouffartigue (Anmerkung 15), S. 381.

31 Smith (Anmerkung 21), S. 143-144, 205-206, sucht den intellektuellen Gehalt der julianischen Schrift herunterzuspielen, aber auch er räumt einen neuplatonischen Einfluss ein. Meine Absicht ist es lediglich, zu zeigen, dass der Kaiser gewisse Argumente auf ganz spezifische Demiurgieauffassungen stützt.

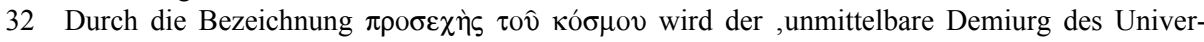
sums` sowohl von den ihm übergeordneten, transzendenteren Göttern, die ein größerer ,Abstand“ von der Welt trennt, als auch von den untergeordneten Göttern, die nicht als Vorgesetzte der ganzen Welt auftreten können, unterschieden. 
zeigt. ${ }^{33}$ Die Tatsache, dass Moses behauptet, der Weltschöpfer habe das jüdische Volk auserwählt, ist ein starkes Indiz dafür, dass der alttestamentarische Gott überhaupt kein universeller Demiurg, sondern lediglich eine lokale Gottheit ist. Welche Götter für die anderen Völker zuständig sind und wie sie ihre Herrschaft gestalten, erwähnt Moses nicht, es sei denn man müsse ihn so verstehen, so Julian, dass er Sonne und Mond als die Götter aller Völker betrachtet. ${ }^{34}$ Letztere Bemerkung bezieht sich auf Deuteronomium 4,19:

Hebe auch nicht deine Augen auf gen Himmel, dass du die Sonne sehest und den Mond und die Sterne, das ganze Heer des Himmels, und fallest ab und betest sie an und dienest ihnen. Denn der Herr, dein Gott, hat sie zugewiesen allen andern Völkern unter dem ganzen Himmel.

Trotz Julians Versprechen erfahren wir in den erhaltenen Fragmenten nichts Genaueres über jene Stelle. Desto ausführlicher aber berichtet er über die Tatsache, dass sowohl Moses als auch Jesus und Paulus (,der Mann, der alle Zauberer und Scharlatane in jeder möglichen Hinsicht überbietet ${ }^{(35}$ ) ihren Gott als den Gott Israels und Judäas betrachten, wobei allerdings Paulus, je nachdem es ihm besser passt, oft seinen Gott als den Gott der Juden, aber manchmal auch als Gott ,der Völker' bezeichnet. Paulus verstrickt sich aber in Widersprüchen. Denn, wenn sein Gott der Gott aller Menschen ist, warum hat er sich nicht um die anderen Völker gekümmert, indem er zum Beispiel seine Propheten auch zu ihnen geschickt hätte? ? $^{36}$

Für Julian ist die Sache klar: dieser Gott ist ein regionaler Gott, mit einem be-

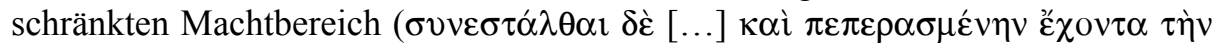

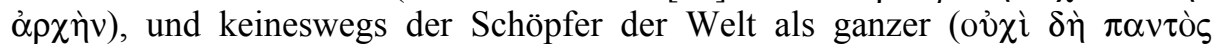

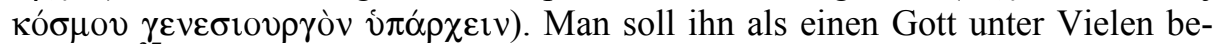
trachten. ${ }^{37}$ Der ,gottlosen' beziehungsweise der jüdisch-christlichen Sichtweise setzt Julian die platonische entgegen, der zufolge man unterscheiden sollte zwischen dem Demiurgen von allem, dem gemeinsamen Vater und König, einerseits, und den Schutzgöttern von Städten und Völkern, die jeweils ein kleineres Gebiet betreuen, ${ }^{38}$ andererseits. Während im universellen Demiurgen alles vollkommen und einheitlich ist, gibt es bei den untergeordneten Göttern eine Zerteilung, ${ }^{39}$ die sich meistens als Spezialisierung äußert, weshalb Ares als Kriegsgott und Hermes

33 Siehe Contra Galileos, Fragment 19,11-12 [99D].

34 Siehe Contra Galileos, Fragment 19,18-19 [99E].

35 Contra Galileos, Fragment 19,23-24 [100A].

36 Siehe Contra Galileos, Fragment 19,20-32 [100A-B]; 20,1-27 [106A-E].

37 Siehe Contra Galileos, Fragment 19,35-37 [100C]. Darüber hinaus argumentiert Julian, dass, auch wenn der mosaische Gott der ,unmittelbar benachbarte', universelle Weltschöpfer wäre, die griechische beziehungsweise die platonische Darstellung dieser Gottheit präziser ist. Siehe Contra Galileos, Fragment 25,9-12 [141D]; 28,2-12 [148B-C].

38 Vergleiche Origenes, Contra Celsum 5,28.

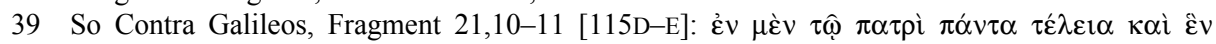

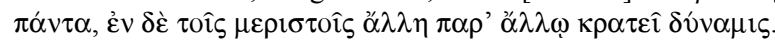


als Gott der Klugheit bekannt sind. Die unterschiedlichen Völker übernehmen dabei den Wesenscharakter der ihnen zugeteilten Gottheit, was eine Erklärung für die diversen Volksarten bietet. ${ }^{40}$

Julian wirft den Juden und Christen also nicht vor, dass ihr Gott kein Schöpfergott sei, sondern dass sie die wahre, lokale und relativ niedrige Natur dieser Gottheit verkennen. Statt sich die Weisheit anderer Völker anzueignen, lehnen sie diese schärfstens $a b$ und beharren in ihrer eigenwilligen, aus Ignoranz entstandenen Weltanschauung.

\section{Helios als Demiurg in platonischer Tradition}

In der Streitschrift Gegen die Galiläer bildet der platonische Gottesbegriff lediglich die Folie, vor der sich Julians Polemik abspielt. Für eine positive und systematische Darstellung von Julians eigenen Auffassungen sind wir dort nicht am richtigen Ort. Mehr Details über Julians Demiurgieauffassung kann man allerdings seinen anderen Werken, insbesondere der Rede auf den König Helios (oratio 11 [4]) sowie der Rede auf die Göttermutter (oratio 8 [5]) entnehmen. ${ }^{41}$

Julian beteuert, seine Helioshymne stelle lediglich den unvollkommenen Versuch dar, die Ansichten des ,göttlichen Jamblich` wiederzugeben, da es nicht möglich sei, Besseres zu diesem Thema zu schreiben als es der vom Gotte geliebte Jamblich getan hat. ${ }^{42}$ Der Einfluss Jamblichs zeigt sich schon daran, dass innerhalb der plotinischen Geisthypostase zwischen einem noerischen (intellektiven) und einem noetischen (intelligiblen) Aspekt als real verschiedenen Bereichen unterschieden wird.

Die sichtbare Sonne ist Jamblich zufolge ein Abbild des noerischen Helios, der selber vom höchsten Gott, dem Einen beziehungsweise Guten hervorgebracht ist. So wie das Eine über den noetischen Bereich und der noerische Helios über die noerischen Götter waltet, so herrscht die Sonne im Bereich des sinnlich Wahrnehmbaren. Ein wichtiges Anliegen der Helioshymne ist es aufzuweisen, wie dem Helios als Prinzip des noerischen Bereichs die zentrale beziehungsweise

40 Hierzu Contra Galileos, Fragment 21,6-29 [115D-116B]. Vergleiche Smith (Anmerkung 21), S. 195-196. Jedes Volk hat nicht nur seinen eigenen Schutzgott, sondern jedem wird zudem jeweils von einem Engel, einem Daimon und einer Seele eines bestimmten Typs assistiert. Siehe Contra Galileos, Fragment 26,2-6 [143A].

41 Ich danke Herrn Gabor Buzasi ganz herzlich dafür, dass er mir freundlicherweise umfassende Materialien aus seiner Dissertation zur Helioshymne zur Verfügung gestellt hat. Für besonders hilfreich halte ich seine scharfsinnige Strukturanalyse der Hymne, sowie seine neue Übersetzung, die wesentlich genauer als die bisher verfügbaren Übersetzungen ist. Wichtige Überlegungen zur Struktur der Hymne findet man auch bei Smith (Anmerkung 21), S. 144-145.

42 Siehe oratio 11 [4], 44,5-9 [157C]. Siehe auch 26,7-11 [146A]; 34,4-6 [150C-D]. Smith (Anmerkung 21), S. 92-113, 135, 139-178, argumentiert auf überzeugende Weise gegen die verbreitete Ansicht, die Heliosrede stelle im Wesen eine mithraische Lehre dar, und betont dagegen die chaldäischen und jamblicheischen Einflüsse. 
die mittlere Stellung zukommt, analog zur zentralen Bedeutung der Sonne für die sinnlich wahrnehmbare Welt. Die noerische Sonne wird mit dem Demiurgen des Universums identifiziert, der demzufolge nicht das höchste metaphysische Prinzip ist. Bildet das Sonnengleichnis aus Platons Politeia eine wichtige Folie für die Helioshymne, so läuft die Annahme einer noerischen Ebene einer unproblematischen Deutung des Gleichnisses zuwider, da jetzt zwangsläufig ein zweistufiges durch ein dreistufiges Modell ersetzt wird. Nur insofern die sichtbare Sonne als eine bloße Manifestation des noerischen Helios und nicht so sehr als eine eigenständige Gottheit aufgefasst wird, könnte Julian dieses Problem einigermaßen vermeiden. Obwohl er in der Hymne deutlich dazu tendiert, die Identität der sichtbaren mit der unsichtbaren Sonne zu betonen, gibt es jedoch auch Stellen, in denen er den Gott Helios und die Sonnenscheibe als eigenständige Prinzipien behandelt. ${ }^{43}$

Auf jeden Fall bildet die Analyse der Verhältnisse innerhalb des Sichtbaren einen wichtigen Zugang zur unsichtbaren Wirklichkeit, deren Struktur uns sonst weithin verschleiert bliebe. Darum bedient sich Julian in der Hymne ständig der analogisierenden Methode, die Rückschlüsse auf eine sonst im Verborgenen liegende Wirklichkeit ermöglichen soll. Im Kern handelt es sich um die exegetische Technik, die im späteren Platonismus unter Berufung auf Platons ,ikonische“ Lehrmethode $^{44}$ auf dessen Texte angewandt wird, damit man sein System rekonstruieren beziehungsweise die Struktur der geistigen Welt aufdecken könne. Platon habe den ,ikonischen Modus' im Sonnengleichnis, ${ }^{45}$ aber vor allem im Timaios weitgehend zur Anwendung gebracht. Genau an der Stelle übrigens, an der Julian auf die Schwierigkeit, über den Gott Helios konkrete Aussagen zu machen, hinweist, liegt eine deutliche Anspielung auf die berühmte einschlägige TimaiosStelle vor. ${ }^{46}$ Julian hält die auf Analogieschlüssen beruhende Verfahrensweise im Vergleich zur exegetischen Lektüre der großen inspirierten Dichter für die überlegenere, da Gott uns durch seine Werke in der Natur direkt belehre, während die

43 Siehe oratio 11 [4], 6,14-18 [133C]. Vergleiche Foussard (Anmerkung 65), S. 205.

44 Der ikonische Modus stellt einen der vier didaktischen tropoi dar. Vergleiche Opsomer, Jan, Deriving the three intelligible triads from the Timaeus, in: Proclus et la Théologie Platonicienne. Actes du Colloque International de Louvain (13-16 mai 1998). En l'honneur de H.D. Saffrey et L.G. Westerink, Hg. A. Ph. Segonds/C. Steel, Leuven/Paris 2000, S. 351-372.

45 In dem von Julian wortwörtlich zitierten Abschnitt aus dem Sonnengleichnis wird ausdrücklich auf die genannten analogen Beziehungen hingewiesen. Siehe 5,18-23 [133A] (= Politeia VI,

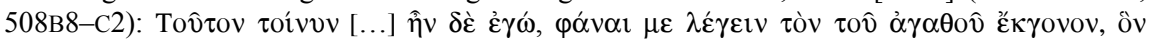

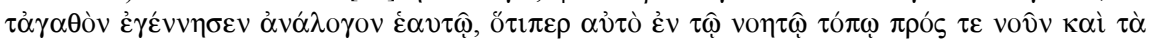

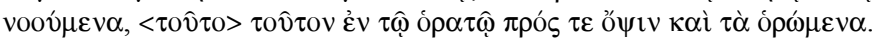

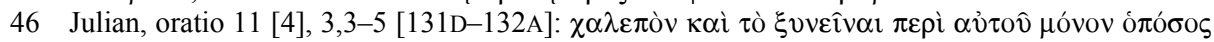

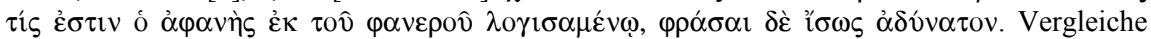

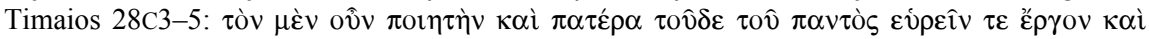

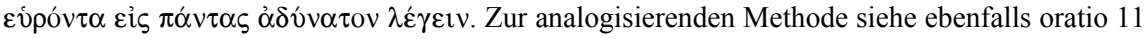

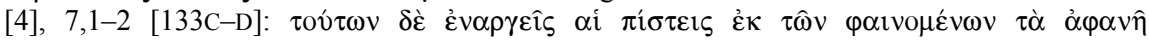

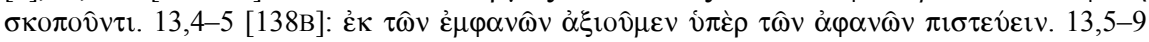
[138B-C]; 35,6-7 [151A] et passim. 
Vorstellungen der Dichter immer auch menschliche Ansichtsweisen in ihre theologischen Ausführungen einmischen. ${ }^{47}$

Gleich im Proömium legt Julian bereits großen Wert auf die Feststellung, dass Helios der Vater aller Menschen ist. ${ }^{48}$ Der Kontrast zum mosaischen Gott fällt sofort ins Auge. Helios ,sät die Seelen der Menschen nicht nur von sich heraus, sondern auch durch die anderen Götter über die Erde aus". Auch wenn die Metapher deutlich an Timaios 41D-E erinnert, bleibt die Art der Beteiligung der anderen Götter zunächst undeutlich. Gemeint ist wohl, dass, während Helios - der mit dem Demiurgen identifiziert wird - die rationale Seele aus sich selbst heraus hervorbringt, die anderen Götter, das heißt die Jungen Götter aus dem Timaios, die einen astralen Charakter haben ${ }^{49}$ für die sterblichen Teile des Menschen verantwortlich sind.

Im Hauptteil der Rede bemüht sich Julian, zum Einem den Ursprung und das Wesen des Helios, zum Anderen seine Macht und Werke in den drei Bereichen seiner Tätigkeit (auf der noerischen, der himmlischen und der sublunaren Ebene) und schließlich seine Wohltaten für die Menschheit aufzuweisen. Dabei wird Helios bald vorgestellt als ein einziger, aber auf verschiedenen Ebenen tätiger Gott ${ }^{50}$ bald als handle es sich um unterschiedliche von einander im UrbildAbbild-Verhältnis abgeleitete beziehungsweise einander hervorbringende Götter, ${ }^{51}$ ohne dass Julian diese beiden Vorstellungsweisen als miteinander inkompatibel zu betrachten scheint.

Die sichtbare Welt bildet eine Einheit, die gemäß der göttlichen Vorsehung von göttlichen Prinzipien ewig erhalten beziehungsweise zusammengehalten wird. $^{52}$ Als angrenzendes $(\pi \rho 0 \sigma \varepsilon \chi \hat{\omega} \varsigma)$ Prinzip gilt „,der fünfte Körper, ${ }^{53}$ dessen

47 Siehe oratio 11 [4], 11,36-39 [137C]. Vergleiche 13,13-14 [138C].

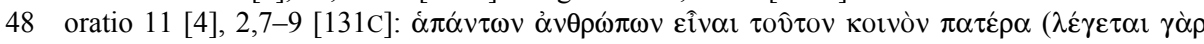

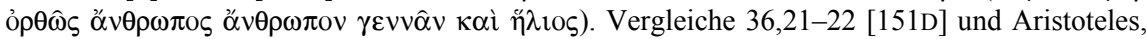

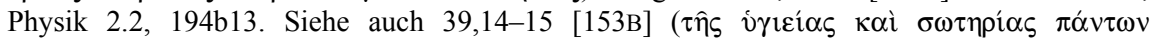

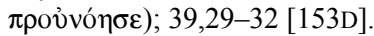

49 Lacombrade (Anmerkung 2), S. 102, Anmerkung 2 (S. 202), weist auf die chaldäische Tradition hin, der zufolge die Seelen in ihrem Abstieg einen astralen Körper erwerben. Nach Jamblichs Ansicht wird das so genannte ,pneumatische Gefährt' von den Göttern selbst hervorgebracht (siehe In Timaeum, Fragment 81 und 84 Dillon). Allerdings gibt es kein Indiz dafür, dass Julian hier eine Beschränkung der demiurgischen Rolle der anderen Götter auf das ,pneumatische Fahrzeug' vorschwebt. Vielmehr hat er wahrscheinlich an alle sterblichen Teile der Menschen gedacht (also auch an die irrationale Seele selbst sowie den Körper beziehungsweise ,das irdische Fahrzeug'). Vergleiche oratio 11 [4], 37,6-9 [152B].

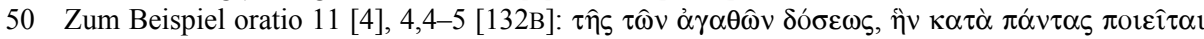

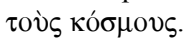

51 Beispielsweise in oratio 11 [4], 5,14-17 [132D-133A]: Helios wird vom Einen und aus dem Einen heraus hervorgebracht und ist ihm ähnlich.

52 oratio 11 [4], 5,2 [132C]. Siehe auch 12,3 [137C]; 15,12-15 [139C-D]; 43,4-14 [156D-157A]. Technisch gesprochen handelt es sich um eine synektische Ursächlichkeit. Siehe hierzu Steel, Carlos, Neoplatonic versus Stoic Causality: The Case of the Sustaining Cause («sunektikon»), in: Quaestio, 2 (2002), S. 77-93.

53 Siehe auch 15,11-14 [139C]. 
Gipfel der Sonnenstrahl bildet“ “54 auf einer höheren Stufe ist die gesamte geistige

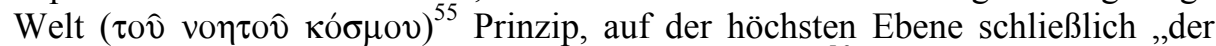
König aller Dinge, um den herum sich alles gruppiert. ${ }^{\text {" } 56}$ Letzterer ist jenseits des Geistes ( $\dot{\pi} \pi \dot{\varepsilon} \kappa \varepsilon \iota v \alpha$ vov̂) gelegen, und kann als ,Idee der Seienden', als ,das Eine“ oder auch als ,das Gute ' bezeichnet werden. ${ }^{57}$ Die Vorstellung eines jenseits des Seienden befindlichen Prinzips (spätestens seit Plotin wird die Seins- mit der Geisthypostase gleichgesetzt), das als das Gute bezeichnet wird (und das im Platonismus mit dem Einen aus dem Parmenides identifiziert wird ${ }^{58}$ und dessen sichtbares Abbild die Sonne ist), sowie die Vorstellung dieses Prinzips als ,König' lassen sich auf das Sonnengleichnis zurückführen. ${ }^{59}$ Die dreistufige Hierarchie beziehungsweise die Vorstellung einer Gruppierung aller auf einer Stufe befindlichen Seienden um ihr Prinzip herum stellt jedoch eine unverkennbare Anspielung auf den (pseudo-platonischen) zweiten Brief dar:

Alles ist um den König aller [Dinge] herum, und seinetwegen sind alle [Dinge], und er ist die Ursache aller schönen [Dinge]. Um den zweiten [König] herum sind die zweiten [Dinge] und um den dritten herum die dritten. ${ }^{60}$

Das höchste Prinzip ist die einheitliche ${ }^{61}$ Ursache aller Dinge, die für jedes Seiende die Quelle von dessen Schönheit, Vollendung, Einheit und Kraft ist. ${ }^{62}$ Kraft

54 Wie sich herausstellen wird, drückt Julian mit diesen Worten aus, dass die Sonne das Prinzip der sichtbaren Welt ist. Vergleiche Raeder, Hans, Kaiser Julian als Philosoph und religiöser Reformator, in: Classica et Mediaevalia, 6 (1944), S. 179-193, hier 210.

55 Die Bedeutung dieses Ausdrucks beschränkt sich nicht auf die noetische Welt im strengen Sinn. Gemeint ist eher die Gesamtheit der noerischen und noetischen Bereiche. Dieser Gebrauch des

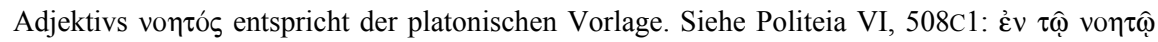

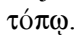

56 oratio $11[4], 5,7-8[132 \mathrm{C}]$.

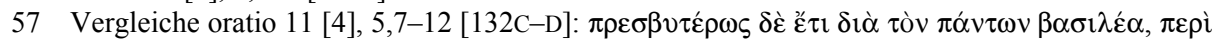

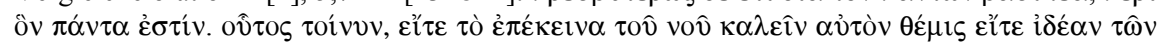

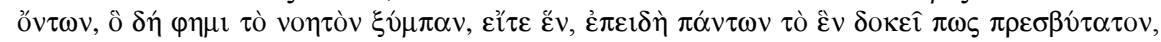

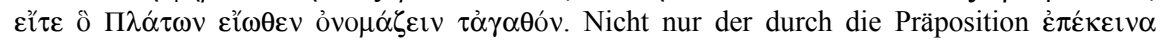
ausgedrückte Transzendenzbegriff, sondern ebenfalls die durch einen Verweis auf die Würde ( $\pi \rho \varepsilon \sigma \beta v \tau \varepsilon \rho \omega \varsigma-\pi \rho \varepsilon \sigma \beta \hat{\tau} \tau \alpha \tau o v)$ markierte Stufung deuten auf das Sonnengleichnis (siehe Anmerkung 59). Vergleiche zudem Numenios, Fragment 17 Des Places.

58 Vergleiche Dodds, E.R., The Parmenides of Plato and the Origin of the Neoplatonic 'One', in: Classical Quarterly, 22 (1928), S. 131-142.

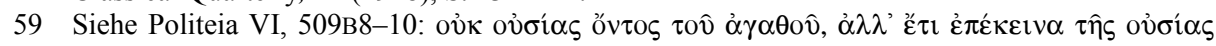

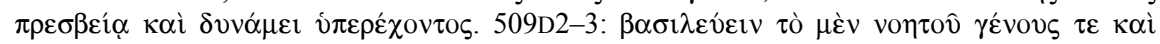

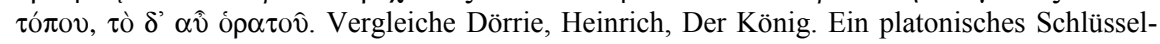
wort, von Plotin mit neuem Sinn erfüllt, in: Revue internationale de Philosophie, 24 (1970), S. 217-235; Mau, Georg, Die Religionsphilosophie Kaiser Julians in seinen Reden auf König Helios und die Göttermutter. Mit einer Übersetzung der beiden Reden, Leipzig/Berlin 1907, S. 30 33.

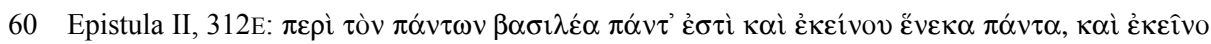

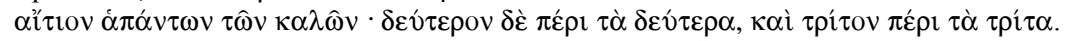

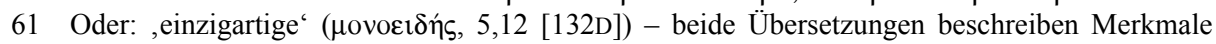
des Einen. 
des in ihm verbleibenden primordialen Seins ${ }^{63}$ bringt es aus sich heraus den ,größten Gott Helios' hervor, der die mittlere Stellung einnimmt unter den mittleren, als ,noerisch ${ }^{6}$ und ,demiurgisch' bezeichneten Ursachen und dem ersten Prinzip in allen Hinsichten ähnlich ist. ${ }^{64}$

Im Anschluss an diese knappe Erörterung der drei ontologischen Stufen ${ }^{65}$ wird der Kernsatz des Sonnengleichnisses zitiert, in dem Platon die Beziehungen zwischen Sonne (dem Sprössling des Guten) und sichtbarer Welt einerseits und dem Guten und dem noetischen Ort andererseits als analog bezeichnet. ${ }^{66}$ Den auf das Zitat folgenden Erläuterungen kann man jedoch entnehmen, dass Julian der Ansicht ist - entgegen dem Wortlaut des zitierten Textes -, Platon meine mit ,Sonne" nicht die sichtbare, sondern die noerische Sonne, das heißt den Großen Helios. Als ein dem Guten ähnliches Prinzip herrscht er über die noerischen Götter, obwohl letztere mit ihm zusammen zeitlos aus dem Einen-Guten hervorgegangen sind ${ }^{67}$ An dritter Stelle schließlich kommt die Sonnenscheibe, die die Welt des Werdens in ihrer Existenz erhält. ${ }^{68}$

Aufgrund der bisherigen Ausführungen Julians kann man zusammenfassend sagen, dass die drei großen ontologischen Bereiche - in absteigender Reihenfolge, der noetische, der noerische und der sichtbare - jeweils von einem der drei hierarchisch geordneten Prinzipien regiert werden: dem Einen-Guten, dem Großen Helios und der sichtbaren Sonne. Diese nehmen innerhalb ihres Bereichs jeweils die höchste Stellung ein, die im Anschluss an Epistula II, 312E die ,mittlere' (beziehungsweise die ,zentrale`) genannt wird. ${ }^{69}$ Zumindest für das erste Prinzip gilt jedoch zugleich, dass es den intelligiblen Bereich übersteigt. Man könnte diese Ambivalenz als Konsistenzmangel bewerten, aber zu Julians Verteidigung kann man anführen, dass er lediglich Platons zweideutige Beschreibung der Idee des

62 oratio 11 [4], 5,13-14 [132D]; ebenfalls 6,7-10 [133B]. Im Gegensatz zu Smiths Behauptung ([Anmerkung 21], S. 148), handelt es sich hier nicht um einen angeblichen noetischen Helios, sondern um das Eine-Gute, was nirgendwo als Helios bezeichnet wird.

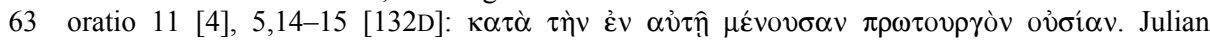
verweist auf das Enthalten-Sein des ersten Seinsprinzips im Einen. Durch diese Ausdrucksweise droht allerdings das höchste Prinzip im Vergleich zu Jamblich an Transzendenz einzubüßen

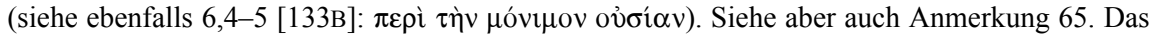

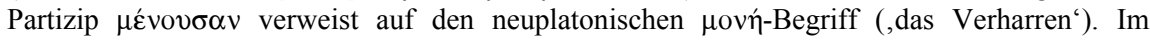

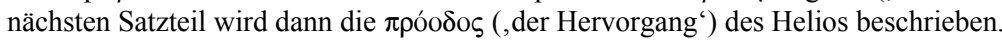

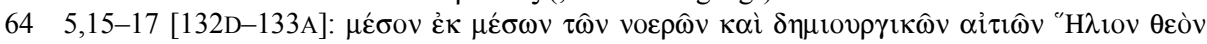

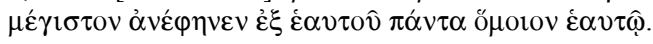

65 Vergleiche Iamblichus, De Mysteriis 8,1-3 (260,3-264,4), wo allerdings ein noch höheres erstes, über das höchste Gut hinausgehendes Prinzip angenommen wird. Siehe Foussard, JeanClaude, Julien philosophe, in: L'Empereur Julien. De l'histoire à la légende (331-1715), Hg. R. Braun/J. Richer, Paris 1978, S. 189-212, hier 203.

66 Vergleiche Politeia 508B8-C2 (zitiert in Anmerkung 45).

67 Siehe oratio 11 [4], 6,1-14 [133B-C].

68 Siehe oratio 11 [4], 6,14-18 [133C].

69 Man denke ebenfalls an Plotins Kreismetapher. Siehe zum Beispiel Enneade I,7 [54] 1,23-26 (mit Bezug auf das Gute, verglichen mit der Sonne als Zentrum des von ihr ausgehenden Lichts); 4,3 [27] 17,12-14; 4,4 [28] 16,20-25; 6,5 [23] 4,20-24. 
Guten repliziert. ${ }^{70}$ Übrigens ist die Königsmetapher besonders dazu geeignet, diesen ambivalenten Status auszudrücken, denn während der König gewissermaßen zum Volk gehört, ist er zugleich über es erhoben, so wie auch die Sonne nach Platons Gleichnis selbst zur sichtbaren Welt gehört, aber gewissermaßen auch nicht, insofern sie nämlich erst die Bedingung der Sichtbarkeit und des Werdens darstellt.

Da Julian die demiurgischen Ursachen auf der noerischen Ebene ansiedelt, liegt es nahe, den Großen Helios ${ }^{71}$ als den universalen Demiurgen zu betrachten. Der sichtbare Kosmos, dessen göttlichsten Teil das fünfte Element darstellt, wird von der Sonne regiert. Mit anderen Worten, die Sonne hat die Funktion eines untergeordneten Demiurgen, der immerhin noch als Demiurg der gesamten Welt gelten darf. ${ }^{72}$

Dieses Bild des demiurgischen, noerischen Helios als Sprössling des ersten Prinzips und Erzeugers der sichtbaren Sonne wird bestätigt im nächsten Teil der Rede, in dem Julian seine Thesen über den Ursprung und das Wesen des Helios mit unterschiedlichen Argumenten zu belegen versucht (7-18 [133C-142B]). Hier müssen nur die für den demiurgischen Charakter des Großen sowie des sichtbaren Helios einschlägigen Textstellen erwähnt werden. Wie bereits angemerkt, besteht Julians wichtigste Verfahrensweise darin, Analogieschlüsse von dem Sichtbaren auf das Unsichtbare zu ziehen. ${ }^{73}$

Als Indiz für die Erhabenheit des Helios führt der Kaiser zunächst an, dass das Sonnenlicht eigentlich eine unkörperliche ${ }^{74}$ Form des aktuell Transparenten ist, wobei das Transparente selbst bereits eine unkörperliche, sich von den körpergebundenen Eigenschaften abhebende Form ist. Als die höchste Erscheinungsform und sozusagen die vollkommenste Frucht und Blüte des Lichts kann man die Sonnenstrahlen bezeichnen. ${ }^{75}$ Julian verweist 7,15-24 [134A-B] auf die Lehre der Phönizier, die die Lichtstrahlen als unbefleckte Kraftäußerung des reinen Nous betrachten, da der Ursprung des Lichtes kein Körper, sondern nur ein Intel-

70 Man vergleiche Politeia VI, 509B8-10 mit VII, 518C9; 526E3-4; 532C5-6.

71 Ich verwende fortan die Ausdrücke ,der Große Helios' und ,der Gott Helios' zur Bezeichnung des noerischen Helios. Julian spricht auch oft von Helios als dem König des Universums (zum Beispiel in 12,19 [138A]; 24,23-24 [145C]; 26,5-6 [145D-146A]; 32,5 [149D]; 40,35-36 [154D]; 43,2-3 [156C]; 44,26-27 [158B]).

72 Man vergleiche Julians Anspielung auf die Sonne als einen dem jüdischen Schöpfergott überlegenen Gott (siehe oben). Den Gedanken der Sonne als eines sichtbaren Demiurgen, der das Abbild eines transzendenten universellen Demiurgen ist, findet man auch in der hermetischen Tradition. Siehe Corpus Hermeticum exc. 21 (Stobaeus 1,41,11 [I p. 293,21-24 Wachsmuth]): oîv

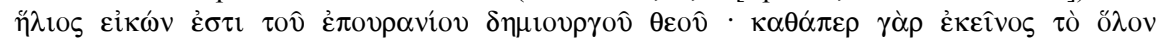

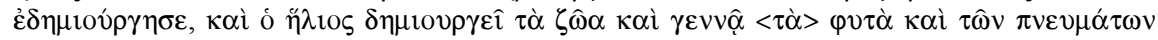
$\pi \rho \tau \tau \alpha v \varepsilon v ́ \varepsilon t$. Diesem Text zufolge ist der transzendente Demiurg der Schöpfer des Ganzen, während die Tätigkeit der Sonne über die unterschiedlichen partiellen Seienden verteilt ist.

73 Siehe zum Beispiel oratio 11 [4], 9,18-28 [135B-C].

74 Siehe auch oratio 11 [4], 16,26-28 [140D].

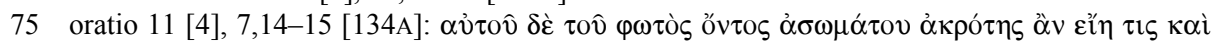

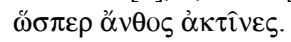


lekt, das heißt der noerische Helios, sein kann. Dieser beleuchte dann die Sonne, die daraufhin das unkörperliche Licht weiter verbreite.

Wie sehr die Eigenschaften des Großen Helios mit denen des sichtbaren Helios verknüpft sind, legen auch die nächsten Argumente nahe. So wie die Sonne die führende Rolle im Kosmos hat, was sich daran zeigt, dass sie die zyklischen kosmischen Prozesse insgesamt verursacht, so regiert der Große Helios unter den überhimmlischen, noerischen Göttern $(9[134 \mathrm{D}-135 \mathrm{C}]){ }^{76}$ Als Merkmale des GroBen Helios lassen sich aus dem Vergleich mit der sichtbaren Sonne insbesondere die folgenden Attribute erkennen: Erstens ist er ein Prinzip der Vervollkommnung ( $\tau$ ò $\mu \grave{\varepsilon} v \tau \varepsilon \lambda \varepsilon \sigma \iota 0 v \rho \gamma o ́ v$ ), weil die sichtbaren Dinge durch das Licht vervollkommnet werden; zweitens ist er ein demiurgisches und zeugendes Prinzip ( $\tau$ ò $\delta \grave{\varepsilon} \delta \eta \mu$ -

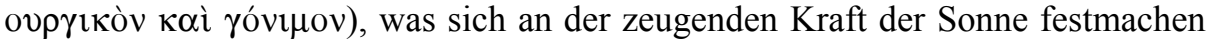
lässt; drittens ist er eine einheitsstiftende, ,erhaltende' beziehungsweise ,synekti-

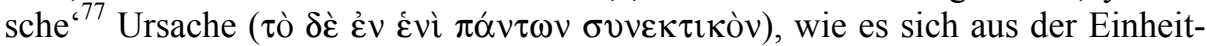
lichkeit und Harmonie der Himmelsbewegungen ergibt; schließlich erkennen wir seine zentrale Stellung ( $\tau$ ò $\delta \grave{\varepsilon} \mu \varepsilon \dot{\varepsilon} \sigma o v$ ), da auch die Sonne die mittlere Position unter den Planeten ${ }^{78}$ einnimmt.

Helios' Herrschaftscharakter wird durch die Identifizierung von Helios mit Zeus bestätigt $(10,10-11[136 \mathrm{~A}]){ }^{80}$ Übrigens ist in der platonischen Tradition die Gleichsetzung von Zeus mit dem universellen Demiurgen geläufig. ${ }^{81}$

Bereits Homer war Helios' führende Stellung unter den noerischen Göttern sowie seine Abstammung vom ersten Prinzip bekannt, wie Julian mit Zitaten nachzuweisen versucht (11 [136B-137C]). Dieser Passus ist auch deshalb interessant, weil er den deutlichen Nachweis dafür liefert, dass Julian es vermeiden möchte, das höchste Prinzip als Helios zu bezeichnen. Der Große Helios ist Julian

76 Siehe insbesondere oratio 11 [4], 9,7-9 [135A]: Die ,überhimmlischen, unsichtbaren und göttli-

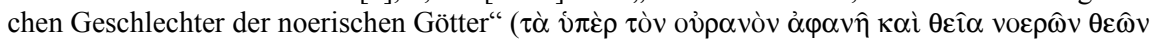

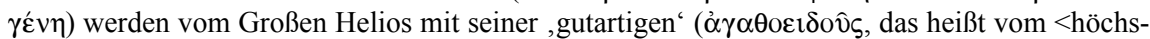
ten> Guten stammenden) Kraft erfüllt.

77 Siehe Anmerkung 52.

78 Es handelt sich um die so genannte chaldäische Reihenordnung: siehe Smith (Anmerkung 21), S. 149. Später erwähnt Julian eine esoterische Tradition, nach der die Sonne nicht in der Mitte der Planeten angesiedelt ist, sondern über die Sphäre der Fixsterne erhoben ist, so dass ihre Mittelstellung in Hinblick auf die drei Welten, nicht auf die der Planeten zu beziehen sei $(28$ [148A-B]). Siehe Lewy, Hans, Chaldean Oracles and Theurgy. Mysticism, Magic and Platonism in the Later Roman Empire, Nouvelle édition par Michel Tardieu, Paris 1978, S. 152-153.

79 oratio 11 [4], 9,26-28 [135C]. Lacombrade (Anmerkung 2), S. 107, Anmerkung 1, weist darauf hin, dass Julian hier die Grenze zwischen dem Großen Helios und dem Himmelskörper verschwimmen lässt. Vermutlich verkennt er dabei die Absicht des Autors, aus den sichtbaren Verhältnissen analogisch auf das Unsichtbare zu schließen.

80 Siehe auch 31,13-14 [149C]. Des Weiteren wird Helios auch mit Apollo und anderen Göttern (für eine Übersicht, siehe Smith [Anmerkung 21], S. 158) identifiziert, was für unser jetziges Anliegen aber weniger wichtig ist.

81 22,12-13 [143D] wird die ,demiurgische Kraft des Zeus' als ein Aspekt des Helios dargestellt. 
zufolge nur der zweite König - eine Tatsache, ${ }^{82}$ die in der Forschung erstaunlicherweise nicht immer so wahrgenommen wird. ${ }^{83}$ Das transzendente Eine ist kein Helios, sondern Helios' Vater und Erzeuger. ${ }^{84}$

Als Demiurg ist Helios Ursache des Entstehens und Vergehens - des Entstehens, indem er sich der körperlichen Natur nähert, des Vergehens, indem er sich wieder entfernt. Damit nicht der Eindruck entsteht, der Gott sei unbeständig, ${ }^{85}$ hält der Autor es für notwendig zu ergänzen, dass die Gabe Gottes an die Welt immer die gleiche sei. Die wechselnde Aufnahmefähigkeit der Empfänger ist es jedoch, die das Mehr und Weniger bestimmt. ${ }^{86}$ So schließt Julian sich dem neuplatonischen Prinzip an, der Grund jeder Schwächung liege immer beim Empfänger, ${ }^{87}$ was damit zusammenhängt, dass transzendente Prinzipien immer nur auf-

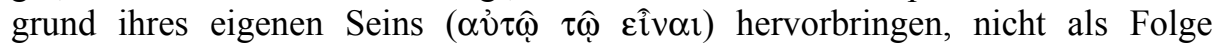
wechselnder von ihnen getroffener Entscheidungen. Denn der Demiurg ist ein Intellekt, ${ }^{88}$ dessen Sein vollständig im Denken besteht, ${ }^{89}$ und zwar in einem über dem Niveau des Diskursiven erhabenen zeitlosen Denken. ${ }^{90}$ Deswegen schöpft er, indem er seine unveränderlichen Gedanken denkt. ${ }^{91}$ Dies ist der Grund, warum Julian behaupten kann, Helios sei sowohl in seiner Substanz als auch in seiner Tätigkeit unveränderlich. ${ }^{92}$ Durch seine ununterbrochene schöpferische Wirkung gewährt er der Welt eine ewige Existenz, die ihr aufgrund ihrer körperhaften Na-

82 Siehe auch oratio 11 [4], 15,20-16,8 [139D-140B].

83 Siehe The Works of the Emperor Julian, Hg. W. C. Wright (Loeb Classical Library), I, Cambridge Massachusetts/London 1913, S. 361, Anmerkung 1; Witt, Rex E., Iamblichus as a Forerunner of Julian, in: De Jamblique à Proclus, Hg. H. Dörrie (Fondation Hardt. Entretiens sur l'antiquité classique, 21), Genève 1974, S. 35-67, hier 38; Lacombrade (Anmerkung 2), S. 86, 107, Anmerkung 1; Proclus, Théologie Platonicienne, Hg. H.D. Saffrey/L.G. Westerink (Collection des Universités de France), Livre II, Paris 1974, S. LVIII; Smith (Anmerkung 21), S. 148 (siehe auch oben, Anmerkung 62).

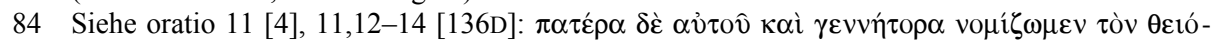

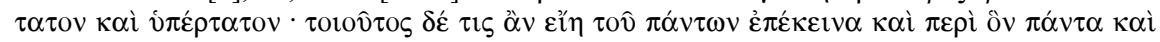

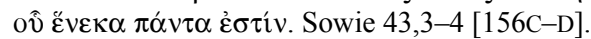

85 Das Sich-Zurückziehen und Sich-Nähern scheint sich vor allem auf die sichtbare Sonne zu beziehen, aber auch in ihrem Fall gilt wohl, dass ihre innere Aktivität unveränderlich ist.

86 Siehe oratio 11 [4], 12 [137C-138A].

87 Siehe zum Beispiel Proclus, Theologia Platonica 1,18, S. 83,24-84,15 Saffrey/Westerink.

88 Vergleiche Opsomer, Demiurges (Anmerkung 25), S. 62, insbesondere Anmerkung 50.

89 Siehe beispielsweise auch Plotin, Enneade 5,1 [10] 8,14-20 (Parmenides Fragment B3).

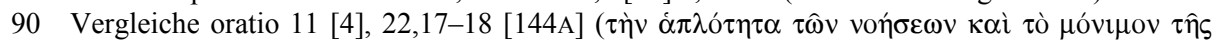

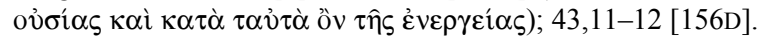

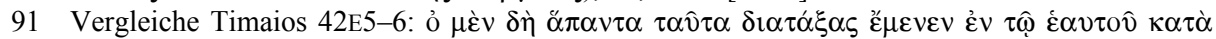

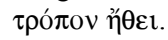

92 Später erläutert Julian, dass für Götter Substanz, Kraft und Tätigkeit identisch sind (20,2-4 [142C-D]), und betont die Unveränderlichkeit der göttlichen Substanz beziehungsweise des göttlichen Willens $(20,14-19$ [143A]). Am sichtbaren Himmel manifestiere sich die Beständigkeit des Helios abbildhaft in der einfachen und unveränderlichen Bewegung der Sonne (12,1920 [138A]). Der Kaiser möchte jedoch nicht leugnen, dass der Wechsel der Jahreszeiten dadurch verursacht wird, dass die Sonne sich nähert und wieder entfernt. 
tur sonst nicht zukäme. ${ }^{93}$ Übrigens ist Helios nicht der einzige noerische Gott, dessen Wirkung wir in unserer Welt spüren. Auch die anderen Intellekte haben einen Einfluss auf die sinnlich wahrnehmbare Welt, aber dank der einigenden Wirkung des Helios gestaltet sich diese Vielfalt von Wirkungen als insgesamt harmonisch, wobei die irdische Harmonie als Zeichen der Verhältnisse in der Geisthypostase betrachtet werden soll. ${ }^{94}$

Die mittlere Stellung des Großen Helios wird zunächst gedeutet im Sinne der ontologischen Verortung des gesamten noerischen Bereiches zwischen der noetischen und der sichtbaren Welt. Helios nimmt somit eine Mittelstellung ein zwischen den noetischen, um das Eine herum gruppierten Göttern, und den ,perikosmischen ${ }^{95}$ Göttern. Unter diesem Ausdruck werden wahrscheinlich die astralen Götter verstanden, einschließlich der Sonne, die den sichtbaren Kosmos umspannen. ${ }^{96}$

In seinem eigenen noerischen Bereich nimmt Helios die zentrale Stellung zwischen den noerischen Göttern ein. Analog zur einheitlichen Ausrichtung des noetischen Bereichs auf das Eine hin, einerseits, und zur synektischen Funktion des fünften Elements, andererseits, hält der Große Helios den noerischen Kosmos zusammen. ${ }^{97}$ In diesem Kontext verweist die mittlere Stellung also auf die vermittelnde, einheitsstiftende Rolle hin, die dem leitenden Prinzip gebührt. ${ }^{98}$ Somit kann man eine doppelte Verwendungsweise beziehungsweise Interpretation des Adjektivs mesos erkennen. Was hierarchisch in der Mitte steht, hat per definitionem nicht die höchste Stellung, während das im Sinne eines einheitsstiftenden, synektischen Prinzips in der Mitte Stehende normalerweise schon die axiologisch höchste Position einnimmt.

Auch als Demiurg nimmt Helios eine mittlere Stellung ein. Man könnte diese Aussage so verstehen, dass der Große Helios sich in der Mitte befindet zwischen dem einen Demiurgen von allem, das heißt dem ersten Prinzip, und den vielen

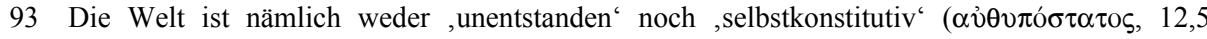
[137D]). Zu letzterem Ausdruck siehe Whittaker, John, The Historical Background of Proclus' Doctrine of the $\alpha \hat{v} \theta v \pi$ ó $\tau \alpha \tau \alpha$, in: De Jamblique à Proclus (Anmerkung 83), S. 193-237. Das Entstandensein der Welt sollte aber nicht so verstanden werden, als sei sie zeitlich entstanden.

94 Siehe oratio 11 [4], 13,5-10 [138B-C]. Vergleiche auch 21 [143B-C]; 22,1-12 [143C-D]. Für die Vorstellung, innerkosmische Götter seien Abbilder noerischer, mit dem Großen Helios zusammenlebender Götter, siehe 23 [144B-C]; 43,10-11 [156D-157A] (

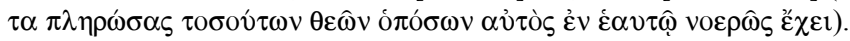

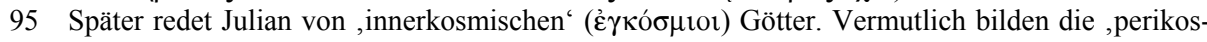
mischen` Götter die höchste Klasse der innerkosmischen Göttern.

96 Siehe oratio $11[4], 13,20-15,14$ [138D-139C].

97 Siehe oratio 11 [4], 15,9-20 [139C-D]. Vergleiche 5,1-2 [132C]; 21,2-3 [143B]; 43,10-12 [156D].

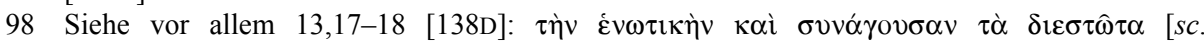
$\mu \varepsilon \sigma o ́ \tau \eta \tau \alpha]$. 
demiurgischen Göttern, das heißt den am Himmel wandernden, astralen Göttern. ${ }^{99}$ Dies wäre überraschend, denn bisher hat Julian die Termini ,Demiurg' und ,demiurgisch“ nur auf den Großen Helios beziehungsweise die noerischen Götter angewendet, und zudem verneinen sowohl Plotin als auch Jamblich und die späteren Platoniker, dass das erste Prinzip als Demiurg betrachtet werden darf. Das Eine ist für sie zwar die erste Ursache, aber keine demiurgische Ursache, da sie dazu tendieren, das Wort ,Demiurg' für die Schöpfung der veränderlichen Welt vorzubehalten, und aus unterschiedlichen Gründen der Ansicht sind, eine solche Tätigkeit wäre für den ersten Gott unangemessen. ${ }^{100}$ Nur die Götter, die die veränderliche Welt hervorbringen, sind demnach demiurgisch tätig; der erste, , universelle' Demiurg wird meistens mit dem Geist beziehungsweise einer Gottheit auf der hypostatischen Ebene des Geistes gleichgesetzt. ${ }^{101}$

Diese Deutung dieser Stelle, derzufolge der Große Helios nur der zweite Demiurg sei, ist jedoch nicht zwingend, wie es eine genauere Lektüre zeigt. Julian sagt zuerst, dass es einen universellen Demiurgen gibt (ohne diesen ausdrücklich zu identifizieren), während die demiurgischen Astralgötter eine Vielheit bilden. Anschließend behauptet er, dass die von Helios ausgehende und sich auf die Welt heraberstreckende demiurgische Kraft als ,ebenfalls in der Mitte zwischen die-

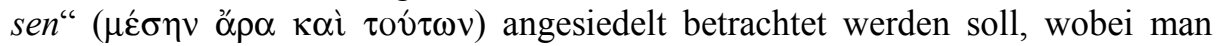
,diese“ als einen Verweis auf die astralen Götter auffassen kann (und nicht als Referenz auf das Eine, einerseits, und die astralen Götter, andererseits). Eine Schwierigkeit für diese Deutung könnte darin bestehen, dass Julian unmittelbar anschließend die lebenerzeugende Kraft des Helios als in der Mitte zwischen der noetischen Lebensquelle und den innerkosmischen Lebensprinzipien liegend beschreibt, so dass man annehmen müsste, dass hier ein neues Argument anfängt, ${ }^{102}$ in dem die mittlere Stellung des Helios nunmehr auf eine strukturell völlig andere Weise gedeutet wird. Für meine Deutung spricht allerdings, dass

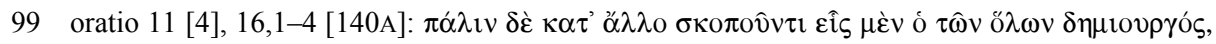

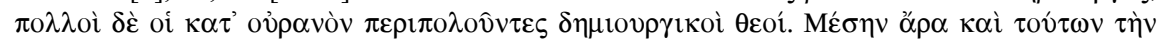

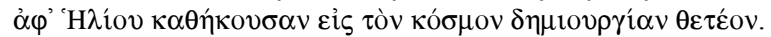

100 Siehe insbesondere Proclus, In Timaeum 1,310,7-15; 1,311,1-4. Zu den philosophiegeschichtlichen Entwicklungen, die zu einer Vervielfältigung der demiurgischen Ebenen sowie zur Herabstufung des Demiurgen geführt haben, zählen der zunehmende Einfluss der aristotelischen Intellektlehre sowie die Kritik am handwerklichen Modell, die in Aristoteles ihren Ausgangspunkt findet. Siehe Opsomer, Demiurges (Anmerkung 25).

101 Bei Plotin gilt grundsätzlich die Geisthypostase als Demiurg, Jamblich identifizierte ihn wahrscheinlich mit einer Gottheit auf einer bestimmten Ebene innerhalb der noerischen Hypostase, während Proklos ihn ganz genau als dritten Gott der ersten noerischen Triade bestimmt. Siehe Opsomer, Jan, Proclus on Demiurgy and Procession in the Timaeus: a Neoplatonic Reading of the Timaeus, in: Reason and Necessity. Essays on Plato's Timaeus, Hg. M.R. Wright, London 2000, S. 113-143; Demiurges (Anmerkung 25); A Craftsman (Anmerkung 27).

102 Dieses Argument würde passenderweise anfangen mit $\dot{\alpha} \lambda \lambda \dot{\alpha} \kappa \alpha \dot{l}$. Jedoch ist die ,zeugende $\mathrm{Kraft}^{\star}$ des Helios so sehr mit seiner demiurgischen Tätigkeit verknüpft, dass Julian das Argument mit der Behauptung beschließt, nichts könne ohne die demiurgische Wirkung des Helios entstehen (siehe 16,10-12 [140B]). 
unverständlich ist, wie Helios die mittlere Stellung einnehmen soll zwischen der Einheit des mit dem ersten Prinzip identifizierten Demiurgen und der Vielheit der astralen Götter, da er selber auch nur einer ist. Aufgrund dieser Überlegungen bleibe ich bei der Ansicht, der Große Helios sei der erste Demiurg. ${ }^{103}$ Auf jeden Fall aber wird deutlich, dass die Demiurgie von Julian, wie von seinen platonischen Geistesgenossen, als ein Prozess zunehmender Zerstreuung und Verteilung aufgefasst wird. ${ }^{104}$ Ausgehend von dem einen, universellen Demiurgen verteilt sich die demiurgische Kraft über immer mehr göttliche Wesen. Dies zeigt sich nicht zuletzt in der Verstreuung der Sonnenstrahlen über die Welt. ${ }^{105}$

Die Wirkung des Großen Helios beschränkt sich selbstverständlich nicht auf die noerische Ebene, auch wenn niedrigere demiurgische Wesen seine Arbeit übernehmen. $\mathrm{Zu}$ seiner schöpferischen Kraft gehört auch, dass er die Prinzipien der sichtbaren Welt in sich trägt. So befinden sich in ihm die Paradigmen der ,Sonnenengel' ( $\tau \hat{\omega} v \dot{\eta} \lambda \iota \alpha \kappa \hat{\omega} v \dot{\alpha} \gamma \gamma \varepsilon \dot{\varepsilon} \lambda \omega v)^{106}$ sowie auch die Ursachen der sinnlich wahrnehmbaren Dinge, sowohl der Himmelskörper wie auch der sublunaren vergänglichen Dinge. ${ }^{107}$ Diese Weise des paradigmatischen Enthalten-Seins der Weltursachen im Wesen des Demiurgen erinnert an Jamblichs Beschreibung der Demiurgie. $^{108}$

Der Unterschied zwischen der himmlischen und der sublunaren Region, der am Ende des ersten Hauptteils der Rede angedeutet wird, spielt eine wichtige Rolle im zweiten Hauptteil der Rede, in dem Julian die Werke und Kräfte des Helios in seinen drei Bereichen erörtert. Diese drei Bereiche sind also die geistige Ebene, die Himmelssphäre und das Gebiet unter dem Mond.

Bei der Behandlung der noerischen Kräfte erwähnt Julian nicht nur den demiurgischen Zeus als einen Aspekt des Helios, ${ }^{109}$ sondern auch, neben andern Göttern, Dionysos. Dionysos wird der Gott der partiellen Demiurgie genannt, der mit Helios fest verbunden, aber dennoch ihm untergeordnet ist. ${ }^{110}$ Während die De-

103 Eine andere Möglichkeit, die Schwierigkeit zu lösen, bestünde darin, Julian eine kleine Inkonsistenz zuzumuten: Er habe sich von den Parallelen mit den anderen Attributen (Einheit und Vollkommenheit; synektische Kraft; Selbstkonstitution; lebenspendende Kraft; Reinheit; Formcharakter) leiten lassen und hier ausnahmsweise auch das erste Prinzip als demiurgisch aufgefasst. Dies entspräche zudem einer platonischen Tradition, nach der zwischen dem Demiurgen der veränderlichen Welt und dem Demiurgen des Seins unterschieden wird und letzterer mit dem höchsten Prinzip gleichgesetzt werden kann. Zum ,Demiurgen des Seins“ siehe Opsomer, Demiurges (Anmerkung 25), S. 52, 64.

104 Siehe oratio 11 [4], 23,8-10 [144C].

105 Siehe oratio 11 [4], 16,35-37 [141A-B].

106 Siehe oratio 11 [4], 17,5-6 [141B]; 18,17 [142A]. Zu diesen gutartigen Gehilfen des Helios siehe Lacombrade (Anmerkung 2), S. 115, Anmerkung 1; Lewy (Anmerkung 78), S. 183, Anmerkung 27.

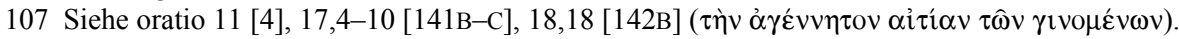

108 Siehe Anmerkung 27.

109 Siehe oratio 11 [4], 22,12-13 [143D].

110 Siehe oratio 11 [4], 22,19-21 [144A]. Siehe auch 29,7-13 [148C-D]. Laut dem Mythos ist Zeus der Vater des Dionysos, wie Julian 152D bemerkt. Siehe auch oratio 8 [5], 19,13-14 [179B]. 
miurgie des Zeus transzendent ist und von der sichtbaren Demiurgie getrennt, überwacht Dionysos kraft seiner ins Einzelne gehenden schöpferischen Tätigkeit die Teilsubstanzen, so wie sie in der sichtbaren Welt vorhanden sind. ${ }^{111}$ In der späteren platonischen Demiurgielehre gilt Dionysos tatsächlich als Schöpfer der partiellen Seienden, was selbstverständlich mit dem Mythos des Zerreißens der Gottheit zu tun hat. ${ }^{12}$ Dionysos' Tätigkeit entfaltet sich Proklos zufolge im Inneren des Kosmos. Julian siedelt ihn hier aber im noerischen Bereich an. Beide Vorstellungsweisen schließen einander jedoch nicht notwendig aus. Zum Einen ist Dionysos auch nach Julian im sichtbaren Kosmos tätig, ${ }^{113}$ zum Anderen gibt es auch bei Proklos Indizien für einen hyperkosmischen Dionysos. ${ }^{114}$ Interessanterweise gibt es eine Stelle in Sallustios' Schrift Über die Götter und die Welt, in der der Verfasser aus der Tatsache, dass Zeus als Weltschöpfer gilt, folgert, dass Dionysos bereits in ihm existieren muss. ${ }^{115}$ Diese Stelle sowie Julians Äußerungen zu Dionysos lassen den Schluss zu, dass die Lehre von Dionysos als partiellem Demiurgen spätestens bei Jamblich zu finden war.

Im Gegensatz zur ,hyperkosmischen' oder ,prokosmischen' Demiurgie wird die Wirkung des Helios auf den himmlischen und sublunaren Bereich ,sichtbar" genannt (die gleiche Unterscheidung findet man auch bei Proklos, der die sichtbare Demiurgie zudem als die ,angrenzende' bezeichnet ${ }^{116}$ ). Helios' Tätigkeit ist hier durch die weltumspannende Sonnenscheibe vermittelt, die als Helios' ,Sitz in unserer Welt betrachtet wird. ${ }^{117}$ Mit ihrer Hilfe vervollkommnet und ordnet er die Welt. ${ }^{118}$ Er tritt unter verschiedenen Aspekten auf, die von den Menschen mit Götternamen versehen werden und die tatsächlich sowohl als von ihm stammende Kräfte wie auch als eigenständige Gottheiten betrachtet werden können ${ }^{119}$ (einige von ihnen erscheinen immerhin auch als Himmelskörper ${ }^{120}$ ). Sofern sie als eigenständige Gottheiten Helios assistieren, gelten sie als Mitursachen der Demiur-

111 Siehe oratio 11 [4], 22,19-28 [144B-C].

112 Siehe Opsomer, La démiurgie des jeunes dieux (Anmerkung 13), S. 17-27; Van Den Berg, Robbert M., Proclus' Hymns. Essays, Translations, Commentary, (Philosophia antiqua 90), Leiden/Boston/Köln 2001, S. 288-292.

$113 \mathrm{Zu}$ seinen Tätigkeiten siehe oratio 11 [4], 29,7-13 [148C-D]; 38 [153B-D].

114 Siehe Opsomer, La démiurgie des jeunes dieux (Anmerkung 13), S. 25.

115 Sallustius, De deis 6,4,5-6. Der Verfasser betrachtet Zeus als enkosmisch, da er (abweichend von Julian, Proklos und anderen) diesen Begriff als eine Bezeichnung für Weltschöpfer aller Art definiert $(6,1,2-3)$.

116 Siehe Proclus, In Timaeum 3,311,21-26; 317,25-28.

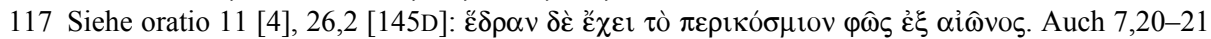

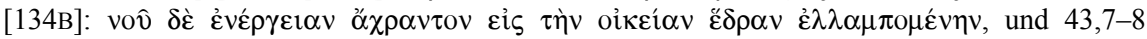
[156D].

118 Siehe zum Beispiel oratio 11 [4], 30 [148D-149A]; 36,1-15 [151A-C].

119 Siehe zum Beispiel oratio 11 [4], 27-29 [147D-148D]; 31-34 [149A-150D].

120 Siehe zum Beispiel oratio 11 [4], 33,5 [150B]. 
gie. ${ }^{121}$ Ihre Tätigkeiten konvergieren aber auf den universellen Demiurgen hin und werden von ihm vereint. ${ }^{122}$

Unter Helios' Tätigkeiten sind besonders hervorzuheben seine Wohltaten für die Einzelseelen, das heißt die menschlichen rationalen Seelen, was schon damit anfängt, dass er ihnen die Lebenskraft verleiht. ${ }^{123}$ Dies entspricht, wie wir bereits gesehen haben, der Darstellung in Platons Timaios. Zudem hilft Helios der Einzelseele bei ihrem Aufstieg zum Göttlichen und hat den Himmel als ,Lehrer der Weisheit' geschaffen. ${ }^{124}$ Obschon der Kaiser gegen Ende seiner Rede den besonderen Bund des Helios mit Rom herausstreicht, lässt er nicht nach, zu betonen, dass Helios der Gott aller Menschen sei. ${ }^{125}$ Dies könnte, wie wir gezeigt haben, als eine verdeckte Anspielung auf den rein nationalen Charakter des jüdischen Gottes gemeint sein.

\section{Zwischenergebnis}

Das sich bisher ergebende Bild der julianischen Demiurgielehre ist komplex, aber dennoch relativ eindeutig. Der Große Helios, Sprössling der ersten Ursache und von deren Gutheit erfüllt, ist der universelle Demiurg. Er wird unter anderem mit Zeus identifiziert, der in der platonischen Tradition mit dem Demiurgen aus Platons Timaios gleichgesetzt wird. Er ist ein Intellekt, dessen Denken zeitlos verläuft, und umfasst in seinem eigenen Wesen sämtliche Ursachen der sichtbaren Welt - die in der Streitschrift gegen die Galiläer erwähnten unsichtbaren Archetypen der sichtbaren Welt. Der Große Helios ist im noerischen (intellektiven) Bereich angesiedelt, das heißt unterhalb der noetischen (intelligiblen) Ebene, und regiert über die noerischen Götter, die mit ihm gleichewig sind und zusammen mit ihm aus dem höchsten Prinzip ,hervorgetreten` sind. Die Tätigkeit des Königs Helios erstreckt sich auf drei Bereiche: auf seinen eigenen noerischen Bereich, auf die Himmelssphäre und auf die sublunare Welt. Wenn Julian am Ende seiner Rede zurückblickend von einer dreifachen Demiurgie des Helios ( $\tau \grave{\eta} \nu \tau \rho \iota \pi \lambda \hat{\eta} v$

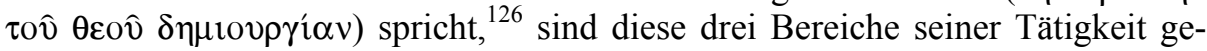
meint - und nicht etwa die drei zentralen ontologischen Prinzipien (das Eine,

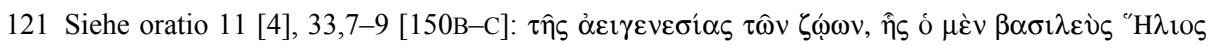

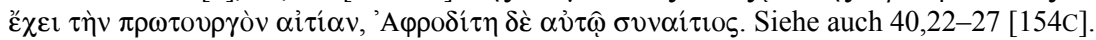

122 Siehe oratio 11 [4], 21,2-3 [143B]; 36,1-15 [151A-C]; 43,10-12 [156D].

123 Siehe oratio 11 [4], 36,16-25 [151C-D]. Vergleiche Timaios 41C6-D1; 41D4-42D5.

124 Siehe Timaios 47A1-B2; 90C6-D7; Epinomis 977A2-B8.

125 Siehe oratio 11 [4], 43,15-16 [157A].

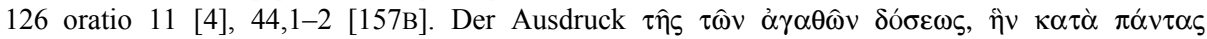

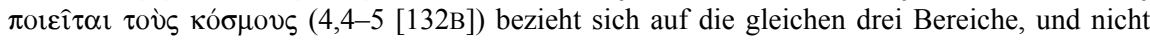
auf die noetische Ebene, den noerischen Kosmos und das sichtbare Universum, wie Wright und auch Smith vermuten (Wright [Anmerkung 83], S. 357-359, Anmerkung 4; Smith [Anmerkung 21], S. 145). 
König Helios, die Sonne), da, wenn ich mit meiner Deutung von 16,3-4 [140A] Recht habe, das Eine von Julian nicht als Demiurg betrachtet wird. ${ }^{127}$

In der sichtbaren Welt ist Helios präsent mittels seines Abbilds, des sichtbaren Helios, der in der Himmelssphäre und aus diesem Grunde auch in der gesamten Welt die zentrale, das heißt die axiologisch höchste Stellung einnimmt und als zwar untergeordneter, aber dennoch universeller Demiurg zu betrachten ist. Auch andere noerische Götter üben eine schöpferische Wirkung in unserer Welt aus, wobei ihre Tätigkeiten durch Helios koordiniert werden. Während sie im noerischen Bereich derart mit Helios vereint sind, dass sie entweder als mit ihm identisch oder zumindest als bloße Aspekte seines Wesens betrachtet werden können, vervielfältigen sich im sichtbaren Kosmos die von ihnen ausgehenden Kräfte wie von einem Punkt aus divergierende Sonnenstrahlen. ${ }^{128}$ Die so genannten astralen Götter sind lediglich sichtbare Abbilder jener noerischen Götter. Sie werden mit den im Timaios erwähnten Jungen Göttern, die von dem ersten Demiurgen erzeugt werden und deren Aufgabe es ist, die Demiurgie auf die sterblichen Teile der Welt auszuweiten, gleichgesetzt. Tatsächlich stehen dem ersten Demiurgen untergeordnete demiurgische Götter zur Seite sowie auch niedrigere Gehilfen, zu denen beispielsweise die Engel gehören. Eine besondere Rolle ist Dionysos zugewiesen, der als Sohn des Zeus-Helios demiurgisch tätig ist und als Prinzip der ,geteilten Demiurgie' gilt. Während Zeus die Welt als Ganzes sowie die universellen Seienden (zu denen der Himmel, die Erde, aber auch die Himmelskörper und die Elemente zählen) zeitlos hervorbringt, übernimmt Dionysos die Verantwortung für die Teile der Welt sowie für einzelne partielle Seiende (diese Rolle des Dionysos ist ebenfalls in der späteren Tradition bezeugt). Allerdings werden die rationalen Teilseelen vom ersten Demiurgen selbst erschaffen.

Die Notwendigkeit untergeordneter Demiurgen lässt sich durch den Intellektcharakter des ersten Helios sowie ebenbürtiger Götter erklären. Da es nicht einzusehen wäre, wie zeitlos denkende Intellekte je innerweltliche Veränderungen verursachen könnten, war es nötig, dass zu diesem Zweck sichtbare, sich bewegende Götter sowie andere Helfer eingeschaltet wurden. Die Demiurgie wird grundsätzlich als ein Prozess der fortschreitenden Aufteilung aufgefasst. Allerdings wird der drohenden Zerstreuung des Seins erfolgreich entgegengewirkt

127 Lacombrade (Anmerkung 2), S. 82, setzt fälschlicherweise die Triade noetisch-noerisch-sichtbar mit einer angeblichen Dreiteilung hyperkosmisch-prokosmisch-sichtbar gleich. Meiner Einsicht nach macht Julian keinen Unterschied zwischen einer pro- und einer hyperkosmischen Ebene, und identifiziert das Hyper- beziehungsweise Prokosmische mit dem Noerischen. Die Gliederung der Abschnitte 21-23 [143B-144C] und 24 [144C-145C] bezieht sich nicht auf einen angeblichen Unterschied zwischen prokosmisch und hyperkosmisch, wie Lacombrade vorgibt, sondern auf einen Unterschied zwischen den Kräften und den Werken des Gottes.

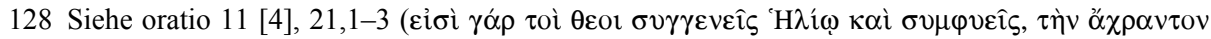

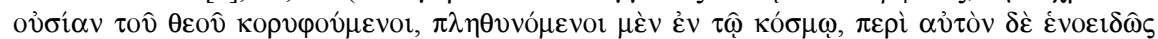

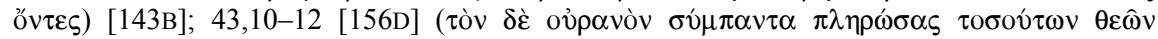

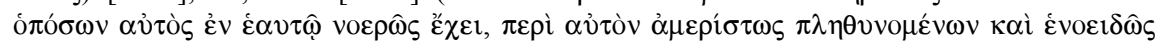
$\alpha \hat{v} \tau \hat{\omega} \sigma v v \eta \mu \mu \varepsilon \dot{\varepsilon} v \omega v)$. 
durch den einigenden, zusammenhaltenden (synektischen) Einfluss des Helios sowie der anderen demiurgischen Götter, der von Julian als eines ihrer wichtigsten Attribute betrachtet wird.

\section{Die demiurgische Funktion von Kybele und Attis}

Ein kurzer Blick auf eine dritte Schrift Julians, die Rede auf die Göttermutter (oratio 8 [5]), soll unser Bild der julianischen Demiurgielehre vervollständigen, wird aber auch neue Probleme aufwerfen. Eine Grundfrage betrifft die Konsistenz der in dieser Rede dargestellten Götter- und Demiurgieauffassungen mit denen der Heliosrede. Julian bestimmt die Göttermutter, Kybele, als Mutter und zugleich Gattin des Großen Demiurgen, das heißt des Großen Zeus, ${ }^{129}$ beziehungsweise des mit Letzterem identifizierten Großen Helios, mit dem sie gemeinsam alles demiurgisch hervorbringt. ${ }^{130}$ Insbesondere ist sie das Prinzip alles Lebens und Entstehens, ${ }^{131}$ sowie, was eng mit der lebenerzeugenden Funktion zusammenhängt, ${ }^{132}$ der fürsorglichen Vorsehung $\left(\pi \rho \rho \mu \eta \dot{\theta \varepsilon i \alpha)}{ }^{133}\right.$ Zudem ist sie die Quelle aller noerischen, demiurgischen Götter, deren Ursachen sie aus dem noetischen Bereich bezieht. ${ }^{134}$

Im Hinblick auf die Helioshymne mag diese Beschreibung des Wesens der Göttermutter einigermaßen überraschend erscheinen, denn dort war nicht nur keine Rede von einer dem Großen Helios gleichrangigen oder sogar höherrangigen demiurgischen Göttin, sondern ebenso wenig wurde die Herkunft der noerischen Götter, einschließlich Helios, mit ihr verbunden. Jetzt aber teilt die Göttermutter Helios' Thron und ist seine Gattin, was sie ihm ebenbürtig zu machen scheint, und es wird behauptet, sie habe ihn und die anderen Intellekte hervorge-

129 Die Hinzufügung der Bestimmung ,Groß` unterscheidet ihn vom gleichnamigen astralen Gott, dem Planeten Zeus (Jupiter).

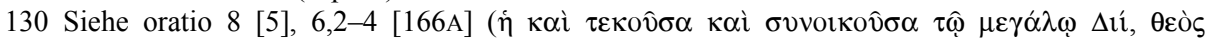

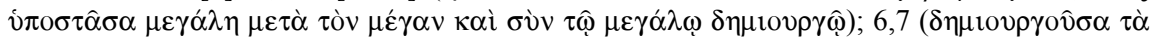

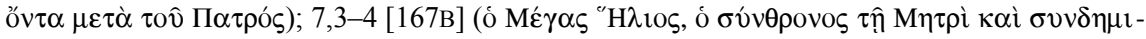

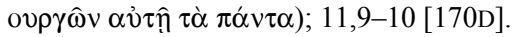

131 Siehe oratio 8 [5], 6,4-6 [166A]; 21,5 [180A].

132 Vergleiche Timaios 30BC; Atticus, Fragment 4,8-13 Des Places = Eusebius, Praeparatio evangelica XV, 6,2; Seneca, Epistula 58,28; Baltes, Matthias, Die Weltentstehung des platonischen Timaios nach den antiken Interpreten, Teil I (Philosophia antiqua 30), Leiden 1976, S. 52-53.

133 Siehe oratio 8 [5], 6,15 [166B] ; 7,4 [167B]; 7,10 [167C]; 7,16 [167D]; 11,7-9 [170D]; 16,6

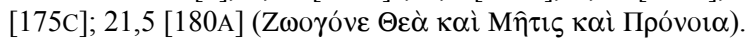

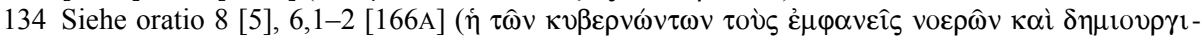

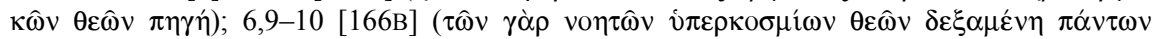

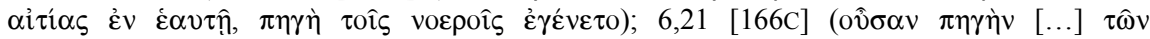

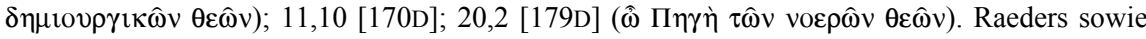
Maus Identifizierung der Göttermutter mit dem höchsten Prinzip ist verfehlt, da Letzteres unmöglich etwas von den noetischen Seienden empfangen könnte. Siehe Raeder (Anmerkung 54), S. 212; Mau (Anmerkung 59), S. 100-101. 
bracht, was eine ontologische Vorrangigkeit impliziert. Wenn sie nun Helios den ersten Platz in der noerischen Welt strittig machen würde, läge eine gewisse Inkonsistenz mit der Heliosrede vor. Allerdings ist meiner Einsicht nach nicht unbedingt klar, ob die Göttermutter überhaupt eine noerische ${ }^{135}$ oder nicht vielmehr eine noetische Gottheit ist. Für letztere Auffassung spricht, dass von ihr behauptet wird, sie bringe alle noerischen Götter als deren Quelle hervor. Dass sie zu diesem Zweck aus der noetischen Substanz schöpft, ist an sich nicht so ausschlaggebend, denn das Gleiche wurde in der Heliosrede vom noerischen Helios gesagt. ${ }^{136}$ Dennoch legt die am Ende der Rede an sie gerichtete Anrede als ,die Du in den Bahnen der unbefleckten Substanzen der noerischen Götter wandelst" ${ }^{\text {137 }}$ die Vermutung nahe, sie sei entweder schlechthin noetisch oder befinde sich mindestens auf der Brücke zwischen beiden Welten (das heißt als eine noerische Göttin, die sich im Noetischen aufhält). ${ }^{138}$

Die Vorstellung, an der Schöpfung, insbesondere an der Zeugung der Lebewesen, sei nicht nur ein männlicher Demiurg, sondern ebenfalls ein weibliches Prinzip beteiligt, findet sich auch in der späteren Tradition, zum Beispiel bei Proklos. ${ }^{139}$ Aber auch in der Heliosrede ist dieser Gedanke nicht abwesend. So werden dort die noerische Göttin Athena, ${ }^{140}$ Aphrodite (der Planet Venus) ${ }^{141}$ und der Mond ${ }^{142}$ im himmlischen Bereich als lebenerzeugende und vorsehende Göttinnen dargestellt, die somit eine weibliche demiurgische Linie bilden. ${ }^{143}$ Dieser Reihe wird in der Rede auf die Göttermutter in der Gestalt der Kybele ein erstes Lebensprinzip vorangestellt. ${ }^{144}$ Die Erörterung des Wesens der Göttermutter ist somit vielmehr als ergänzend zur Heliosrede, denn als mit ihr widersprüchlich zu betrachten. Dies gilt ebenfalls für die Behauptung, die Göttermutter sei die

135 Bei Proklos nimmt Rhea die zweite Stellung innerhalb der ersten noerischen Triade ein. Aber er referiert ebenfalls eine orphische Lehre, nach der die gleiche Göttin, wenn sie ,oben“ ist, ,Rhea“ genannt wird, und, wenn sie bei Zeus ist, ,Demeter'. Siehe In Cratylum 141,4-7; 167,2-4.

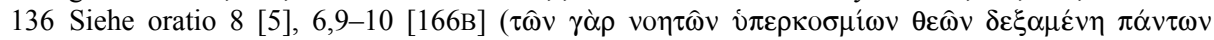

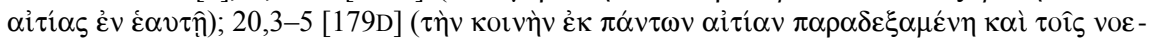

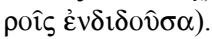

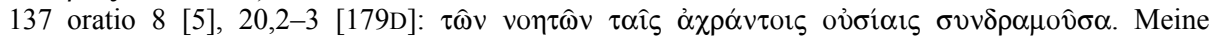
Übersetzung richtet sich nach Asmus, Rudolf, Kaiser Julians Philosophische Werke (Philosophische Bibliothek 116), Leipzig 1908.

138 Vergleiche Wright (Anmerkung 83), S. 440 und 463, Anmerkung 3; Athanassiadi-Fowden, Polymnia, Julian and Hellenism. An Intellectual Biography, Oxford 1981, S. 143; Smith (Anmerkung 21), S. 160.

139 Siehe Theologia Platonica 5,11, S. 39,10-15 Saffrey/Westerink; In Timaeum 3,188,20-28.

140 Siehe oratio 11 [4], 31-32 [149B-150A]. Vergleiche oratio 8 [5], 19,9-11 [179A-B].

141 Siehe oratio 11 [4], 33 [150B-C].

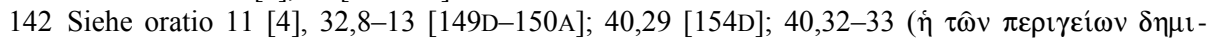

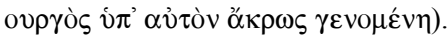

143 Vergleiche Sallustius, De deis 6,3,2.

144 Auch diese Vorstellung findet man in der späteren Tradition, wobei das weibliche Lebensprinzip als $\delta$ v́ $\alpha \mu \iota \varsigma$ typischerweise die Prohodos und das männliche demiurgische Prinzip die intellektive Epistrophe symbolisiert. 
Demiurgin unserer Seelen, ${ }^{145}$ die keineswegs die Verneinung der Heliosvaterschaft impliziert. Trotz der Figur der Göttermutter wird der Helios weiterhin als der Große beziehungsweise universelle Demiurg bezeichnet. Auch Dionysos behält seine Funktion als Haupt der partiellen Demiurgie. ${ }^{146}$

Die Rede auf die Göttermutter ist aber vor allem deshalb interessant, weil sie uns wichtige Einblicke in die niedrigeren Stufen des demiurgischen Geschehens verschafft. Die wichtigste Figur der Hymne ist zweifelsohne Attis, der Geliebte der Göttermutter, der sich aber in eine Nymphe verliebt, daraufhin entmannt wird und sich später wieder mit der Göttermutter versöhnt. ${ }^{147}$ Attis wird von Julian verstanden als „diejenige Substanz des zeugenden und demiurgischen Intellekts, die bis zur letzten Materie hinunter alles erzeugt und in sich alle Prinzipien und

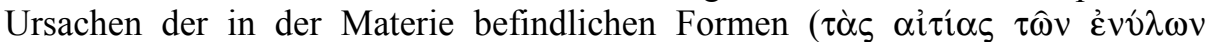

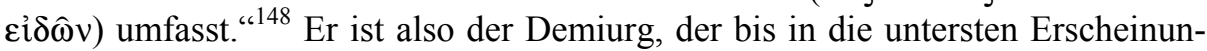
gen der Materie vordringt und sie mit Hilfe formaler Prinzipien ordnet. Die letzten Formen (Ideen) sind die so genannten enhyla eidê, die zusammen mit der unterliegenden Materie die hylemorphischen Körper bilden. Nach der platonischen Sichtweise, bilden sich derartige Zusammensetzungen normalerweise nicht zufällig. Materie und Form bedürfen einer causa efficiens, die sie zusammenführt. ${ }^{149}$ Die demiurgische Kraft, die dies auf der untersten Stufe bewirkt, heißt Attis. Die Formen bezieht er aus den oberen Ebenen, die Materie stellt Julian als vorhanden dar. ${ }^{150}$ Julian erklärt auch, warum Attis, und beispielsweise nicht Helios, die untersten Formen der irdischen Körper hervorbringt: Der Grund ist, dass nicht alle Formen in allen demiurgischen Prinzipien enthalten sind; die partikulären Formen der untersten Seienden sind nicht auf der Ebene der noerischen Götter, sondern nur bei den niedrigeren demiurgischen Göttern vorhanden. ${ }^{151}$ Die universelleren Formen gibt es in den höheren Prinzipien, während einzelne Teilformen erst in den niedrigeren Demiurgen in Erscheinung treten.

Julian wiederholt mehrmals, dass Attis bis in die untersten Regionen der materiellen Welt absteigt, weshalb er als letzter Gott gilt. An einer Stelle äußert er

145 Siehe oratio 8 [5], 20,5-6 [180A].

146 Siehe oratio 8 [5], 19,11-16 [179B]. Maus Argument für die Identität des Attis und des Dionysos ist nicht überzeugend. Siehe Mau (Anmerkung 59), S. 114.

147 Siehe Van Den Berg (Anmerkung 112), S. 171-172; Bouffartigue (Anmerkung 15), S. 376-377.

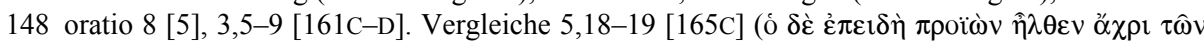

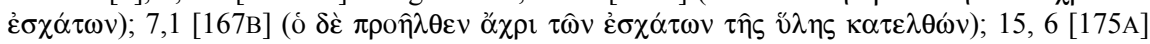

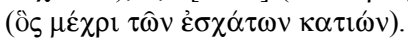

149 oratio 8 [5], 3,17-23 [162A]. Julian polemisiert ausdrücklich gegen die Epikureer.

150 Die Materie ist gleichewig mit den Göttern, entsteht zeitlos ,,aus ihnen und durch sie aufgrund der Fülle der zeugenden und demiurgischen Ursache“ (oratio 8 [5], 11,6-8 [170C-D]). Welche Götter genau die Materie hervorbringen, lässt der Verfasser aber im Dunklen. Allerdings identifiziert er, wie Plotin, die Materie mit Privation (3,11-12 [161D]). Der Ausdruck $\tau \hat{\eta} \varsigma$ ט̋ $\lambda \eta \varsigma$

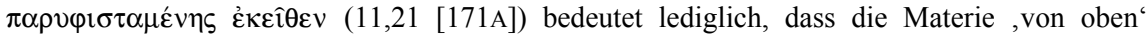
kommt, nicht dass sie an der äußersten Grenze der fünften Substanz aus der Mischung des $\dot{\alpha} \pi \alpha \theta \varepsilon \dot{\varepsilon} \varsigma$ und $\pi \alpha \theta \eta \tau$ óv entsteht, wie die Übersetzungen von Wright und Rochefort suggerieren.

151 Siehe oratio 8 [5], 3,9-11 [161C-D]. 
sogar Zweifel am göttlichen Charakter des Attis, und fragt sich, ob es nicht angemessener wäre, ihn als Halbgott zu bezeichnen. Dennoch hält Julian an der Göttlichkeit des Attis fest. Sein niedriger Status innerhalb der Göttergemeinschaft hindert ihn nicht daran, die so genannten ,Göttlichen Geschlechter' zu dirigieren, die ihm alle unterstellt sind. ${ }^{152}$ Diese Geschlechter, die im Attismythos durch die Korybanten symbolisiert werden - so Julian -, sind Jamblich zufolge die Engel, Heroen und Dämonen. Übrigens lehrt auch Proklos, dass diese als Gehilfen den demiurgischen Göttern zur Seite stehen. ${ }^{153}$

Julian referiert eine alternative peripatetische Lösung des Problems der hylemorphischen Körper, die darin besteht, das Zusammenkommen von Materie und Form durch die Wirkung der ,fünften Substanz ${ }^{`}$ zu erklären. Er hält diese Lösung aber für unzureichend, was noch einmal - so Julian - bestätige, dass trotz des manchmal vernünftigen Ansatzes die aristotelische Philosophie meistens keine befriedigenden Lösungen anzubieten vermag, solange sie nicht durch die platonische ergänzt werde. ${ }^{154}$ Julian untersucht, wie die fünfte Substanz die Ursachen der enhyla eidê enthalten könnte. Dass es diese Ursachen geben muss, ist klar: Woher würden sonst die Arten sowie das männliche und weibliche Geschlecht stammen, wenn nicht die Ursachen dieser formalen Bestimmungen vorher irgendwo als Paradigmen angesiedelt wären? ${ }^{155}$ Der fünfte Körper kann aber nicht den Ursprung der in der Materie befindlichen Formen bilden, da er selber hylemorphisch zusammengesetzt zu sein scheint. ${ }^{156}$ Julian weist darauf hin, dass Aristoteles, nach den Vorgaben Platons, lehre, dass die Seele der Ort der Formen ist, da unser Intellekt (ó vôิs) die Formen der Dinge auf unkörperliche Weise in sich aufnehmen kann. ${ }^{157}$ Die Seele hat die Formen der Dinge aber nur potentiell, wie es auch für die hierarchisch niedrigere Natur gilt. Diese Bemerkung gilt sowohl für die Teilnatur beziehungsweise Teilseele, wie für die universelle Natur beziehungsweise die universelle Seele. ${ }^{158}$ Die Formen müssen jedoch vorher in Aktualität existieren, und dies kann nur der Fall sein in einem reinen Intellekt, nämlich in Attis.

152 Siehe oratio 8 [5], 8,1-12 [168A-B]; 4,28-29 [163C]. Siehe auch Contra Galilaeos, oben, Anmerkung 23 und 40.

153 Siehe Proclus, In Timaeum 1,137,7-26; 310,24-6; In Alcibiadem 68,16-17.

154 Siehe oratio 8 [5], 3,23-40 [162A-D]. Die Bewertung der aristotelischen Philosophie in der Tradition des Platonismus ist Gegenstand einiger neuer Veröffentlichungen: siehe Karamanolis, George E., Plato and Aristotle in Agreement? Platonists on Aristotle from Antiochus to Porphyry, Oxford 2006; Gerson, Lloyd P., Aristotle and other Platonists, Ithaca 2005.

155 Siehe oratio 8 [5], 4,1-8 [162D-163A].

156 Siehe oratio 8 [5], 4,68-69 [165A]. Der schwierige Passus 11,19-21 [171A] ist nicht so zu verstehen, als sei die fünfte Substanz unkörperlich und materiefrei. Er impliziert nur, dass sie

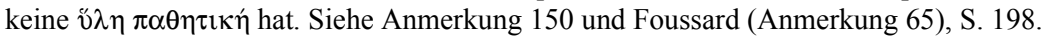

157 Siehe oratio 8 [5], 4,11-17 [163A-B]. Siehe Aristoteles, De Anima 3,4, 429a22-29 (siehe Aristotle, Aristoteles, De anima, Hg. David Ross, Oxford 1999, S. 292); Platon, Theaitetos

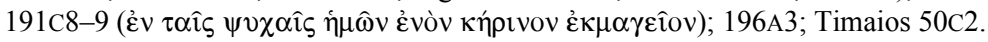

158 Siehe oratio 8 [5], 4,50-67 [164B-D]. 
Die unterschiedlichen Elemente des Attismythos erfahren in Julians Hymne eine allegorische Deutung. ${ }^{159}$ Attis wuchs auf am Rande des Flusses Gallos. Diese Heimat wird von Julian als die ,Galaxie', das heißt die Milchstraße gedeutet, die sich genau an der Grenze zwischen der unaffizierbaren fünften Substanz ${ }^{160}$ und der Welt des Werdens befindet. Attis' Affäre mit der Nymphe und sein Verbleib in der Nymphenhöhle symbolisieren seinen Abstieg in die Welt der Materie, die einen gewissen Reiz auf ihn ausübt. Die Nymphe stehe dabei nicht für die Materie selber, sondern für die niedrigste unkörperliche Form oberhalb der Materie, die des Feuchten. Die Göttermutter wolle aber, dass Attis, den sie wegen seiner Schönheit beziehungsweise seiner demiurgischen Kraft leidenschaftslos liebt, zu ihr zurückkehrt und ,im Geistigen zeugt ${ }^{6}{ }^{161}$ Dieses narrative Moment symbolisiere die epistrophê, durch die Helios gestärkt werden soll. Da Attis' Abstieg nicht ins Unbegrenzte (in die $\alpha \dot{\alpha} \varepsilon \imath \rho^{\prime} \alpha$ ) weitergehe, schicke der Große Helios, Gatte der Göttermutter, einen feurigen Löwen, der Attis dem feuchten Element, das heißt der Nymphe, strittig machen soll. So diene der Löwe der schöpferischen Providenz beziehungsweise der Göttermutter. Die durch dessen Botschaft veranlasste Kastration bedeute, dass Attis' Hang zum Unbegrenzten Einhalt geboten wird. ${ }^{162}$ In den demiurgischen Kontext übersetzt, werde damit die mittels Formen vollzogene ${ }^{163}$ Begrenzung des Unbegrenzten bezeichnet - oder mit anderen Worten, das durch eine vernünftige effiziente Ursache überwachte Entstehen hylemorphischer Substanzen. ${ }^{164}$ Dass dies ohne die aus Vermessenheit begangene Grenzüberschreitung nicht möglich gewesen wäre, zeige wiederum die Unverzichtbarkeit dieses niederen Demiurgen. ${ }^{165}$ Überdies erfülle er aufgrund seiner eigenen Rückkehr (epistrophê) eine anagogische Rolle für die menschlichen Seelen, indem er sie in die Richtung der höheren Prinzipien begleite. ${ }^{166}$ Attis' mit Sternen besetzte Krone weise auf seine Herrschaft, bei der er die Zuständigkeiten der astralen Götter zum Ausgangspunkt mache. ${ }^{167}$

159 Siehe oratio 8 [5], 5-7 [165B-D; 166B-167D]; 11 [170C-171D]; 15 [175A-B]. Der Mythos erfährt eine sehr ähnliche Deutung bei Sallustius, De deis 4,7-9.

160 Vergleiche oratio 8 [5], 11,1-5 [170C]. In diesem Grenzbereich könne man öfters gewisse Änderungen des Mondlichts beobachten, wo die Welt des Werdens der unveränderlichen fünften Substanz näher kommt und die beiden Welten sich mischen: siehe 7,20-25 [167D-168A].

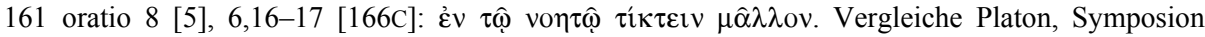
206B7-E5; Iulianus, Contra Galileos, Fragment 10,9-11 [65B] (siehe oben, Anmerkung 15); oratio 11 [4], 24,7-27 [144D-145C].

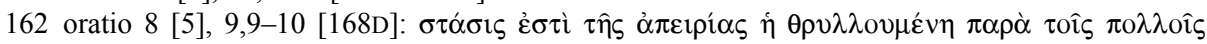

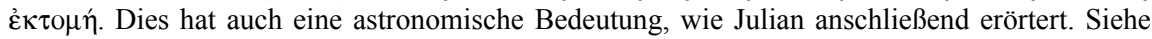
auch 9,33-34 [169C].

163 Als causa efficiens gilt die demiurgische Bewegung des Helios: oratio 8 [5], 15,6-7 [175A].

164 Vergleiche Platon, Philebos 23C9-E6; 25C8-D3; 26C5-27B2; siehe insbesondere 28D5-E6 und

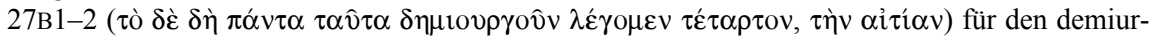
gischen Kontext.

165 Siehe oratio 8 [5], 7,16-20 [167D].

166 Siehe oratio 8 [5], 12,14-33 [172B-173A]; 15,12-13 [175B].

167 Siehe oratio $8[5], 7,16-20[170 \mathrm{D}-171 \mathrm{~A}]$. 
Attis ist der letzte Demiurg-Gott, der bis in die unteren Regionen der Materie hinabsteigt und für das Entstehen und Vergehen der hylemorphischen Körper zuständig ist. ${ }^{168}$ Daher ist er der , angrenzende Demiurg der materiellen Welt' (ó

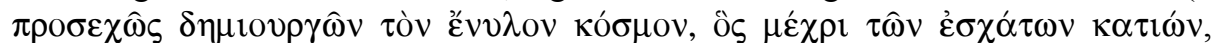
15,5-6 [175A]), womit wohl gemeint ist, dass er der angrenzende Demiurg der sublunaren Welt ist. Da sein eigentlicher Aufenthaltsort, die Milchstraße, die unterste Grenze der himmlischen Region beziehungsweise der fünften Substanz bildet, ${ }^{169}$ dürfte er wohl nicht für den Demiurgen des sichtbaren Himmels, geschweige denn für den angrenzenden Demiurgen des gesamten Universums gehalten werden. Letzterer muss also wohl der sichtbare Helios sein, dessen Reich sowohl den sichtbaren Himmel als auch das Sublunare umfasst.

Hinsichtlich Julians Kennzeichnung des Attis als eines Demiurgen des Werdens und Vergehens ist es interessant zu beobachten, dass der Kaiser einige Passagen aus den platonischen Dialogen in Erinnerung ruft - sei es ohne den $\mathrm{Zu}$ sammenhang zu erläutern -, in denen Platon von ,sophistischen Demiurgen“ spricht. Damit sind Personen gemeint, die sich mit Abbildungen beschäftigen, und mit großer Leichtigkeit eine ganze Welt in einem Spiegel erzeugen können. ${ }^{170}$ Es handelt sich um eine als Spiel aufgefasste mimetische Demiurgie, deren Früchte äußerst flüchtig und ephemer sind, und, da sie nur Spiegelungen sind, den Objekten des untersten Linienabschnittes entsprechen. ${ }^{171}$ Sowohl im Sophistes als auch im zehnten Buch der Politeia ist von einer solchen Scheinkunst die Rede. ${ }^{172}$ Interessanterweise hat gerade Jamblich in seinem Kommentar zum Sophistes den skopos dieses Dialogs als den sublunaren Demiurgen bestimmt (Sophistes-Kommentar, Fragment 1,1-2 Dillon). Dieser beschäftige sich - genau wie auch Attis gemäß Julians Darstellung - mit der Herstellung materieller Dinge (Zeile 6), sei von der Materie gereizt, aber betrachte trotzdem das wahrhaft Seiende (Zeile 7); er imitiere sowohl den himmlischen Demiurgen wie auch den

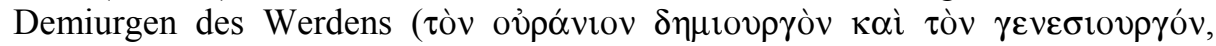
Zeilen 14-16).

Die von Jamblich erwähnte sublunare, ,sophistische‘ Demiurgie könnte mit Proklos’ ,Demiurgie des Adonis“ identisch sein. An einigen Stellen behauptet Proklos nämlich, der dritte Demiurg, nach Zeus und Dionysos, sei Adonis. ${ }^{173}$

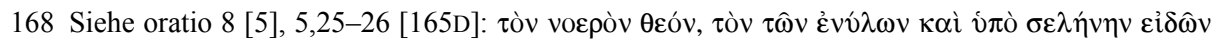

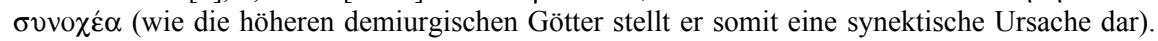

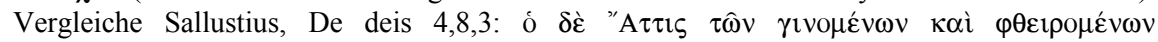

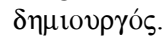

169 Die fünfte Substanz wurde in der ,Heliosrede“ als angrenzendes Prinzip der sublunaren Welt bezeichnet: oratio 11 [4], 5,5 [132C], siehe oben.

170 Siehe oratio 8 [5], 4,22-66 [163C-D].

171 Siehe das Liniengleichnis: Politeia VI, 509E10-510A3; E2-3. Vergleiche auch das Höhlengleichnis, Politeia VII, 515A5-8; C2 (Schatten in der Höhle); 516A7 (Schatten in der Außenwelt).

172 Sophistes 233D9-236C7, insbesondere 136A4-5; 264C-268D; Politeia X, 596C3; D1-E2.

173 Siehe In Timaeum 1,144,5-13; In Rempublicam 2,8,15-23. 
Auch Platon habe dies suggeriert, behauptet Proklos, ohne zu sagen, wo dies der Fall sein soll. Die einzige Stelle, die in Betracht kommt, ist Phaidros 276B, wo Platon das Schreiben mit dem Säen in den Töpfen des Adonis vergleicht. Dort säe man nur aus Spaß, da das Saatgut zwar in acht Tagen schön aufgeht, aber auch schnell wieder verwelkt. Dies ist genau das, was der sublunare Demiurg den Neuplatonikern zufolge bewirkt. Einen weiteren Hinweis findet man in Proklos' Hymne auf Helios, wo Proklos schreibt, Helios manifestiere sich ,im Tiefsten der Materie' als Attis oder, wie andere es meinen, als Adonis. ${ }^{174}$ Diese Zusammenhänge legen die Vermutung nahe, dass das Lehrstück, demgemäß Attis der sublunare Demiurg des Entstehens und Vergehens ist, bereits von Jamblich stammte und sowohl Julian als auch Sallustios inspiriert hat.

Attis hat - so Julian - eine noerische Natur, ${ }^{175}$ die seine Tätigkeiten in den sublunaren Bereich hinein entfaltet. Dies bedeutet wahrscheinlich, dass er, ähnlich wie Helios, ein noerischer Gott mit einem sichtbaren Abbild ist - wobei Julians Rede vornehmlich dem innerkosmischen Attis gilt. Die Frage seiner hierarchischen Stellung lässt sich nicht so leicht beantworten. Mehrmals ist von einem dritten Demiurgen die Rede. Das erste Mal scheint Julian Attis schlechthin mit diesem dritten Demiurgen, „der die transzendenten Begriffe der enhyla eidê sowie die damit verbundenen Ursachen besitzt", oder zumindest mit dessen letzter Natur, „die von den Sternen oben bis unten zur Erde reicht“, zu identifizieren. ${ }^{176}$ Zwei weitere Passagen zeigen, dass die zweite Deutung die richtige ist, und zwar in dem Sinne, dass Attis als ,letzte Natur' dieses dritten Demiurgen aus Letzterem hervorgegangen ist und eine eigenständige Gottheit bildet. ${ }^{177}$ Denn einmal heißt es, dass wir Attis von diesem dritten Demiurgen abtrennen, ${ }^{178}$ ein andermal, dass er aus dem dritten Demiurgen hervorgeht. ${ }^{179}$ In der ersten der genannten Passagen vernehmen wir über den dritten Demiurgen, dass er Vater und Herr nicht nur der enhyla eidê, sondern ebenfalls ,des sichtbaren und fünften Körpers“ ist. ${ }^{180}$ Wer ist also dieser dritte Demiurg? Die meisten Interpreten sind der Auffassung, es handle sich um Helios, mit dessen Strahlen Attis tatsächlich identifiziert wird. ${ }^{181}$ Der

174 Proclus, Hymnen 1, 25-26. Siehe auch Damascius, In Parmenidem 214,5-6: oîov ó "A $\tau \tau \iota \varsigma$ çv

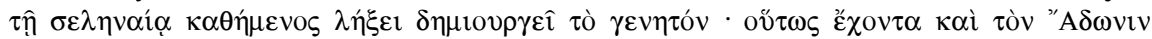

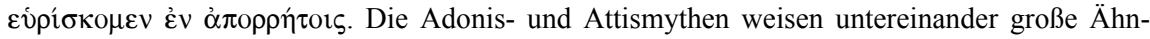
lichkeiten auf. Für eine ausführlichere Erörterung der Adonisdemiurgie siehe Opsomer, La démiurgie des jeunes dieux (Anmerkung 13), S. 38-45.

175 Siehe oratio 8 [5], 3,6 [161C]; 5,18; 25 [165C-D].

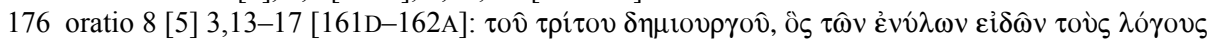

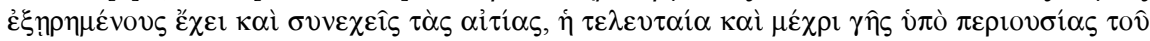

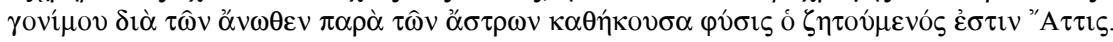

177 Siehe auch die in der Helioshymne dargestellte phönizische Lehre: oben, S. 139-140.

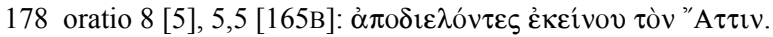

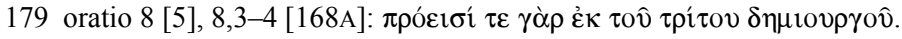

180 oratio $8[5], 5,2-4[165 \mathrm{~A}]$.

181 Siehe Mau (Anmerkung 59), S. 93; Raeder (Anmerkung 54), S. 212; Wright (Anmerkung 83), S. 451, Anmerkung 3; Rochefort (Anmerkung 2), S. 95, 107, Anmerkung 2. Die von Mau (S. 
sichtbare Helios könnte tatsächlich der Demiurg sein, der die Ursachen aller Dinge in sich birgt, denn das wurde auch vom Großen Demiurgen behauptet. Helios könnte auch sehr wohl als Vater des fünften Körpers betrachtet werden. Eine Schwierigkeit scheint aber zu sein, dass er als dritter Demiurg bezeichnet wird. Wenn unsere Interpretation der Heliosrede richtig ist, wird die sichtbare Sonne dort als zweiter Demiurg betrachtet. Liegt hier eine Inkonsistenz vor? Wenn hier die sichtbare Sonne als dritter und die unsichtbare als zweiter Demiurg betrachtet wird, wer ist dann der erste Demiurg? Man könnte vermuten, dieser wäre das Eine-Gute, ${ }^{182}$ was entweder bedeuten würde, dass unsere Interpretation dieses Aspekts der Heliosrede verfehlt ist, oder, dass Julian in der Rede auf die Göttermutter nicht die gleiche Ansicht vertritt. Es scheint aber unwahrscheinlich zu sein, dass in der Rede auf die Göttermutter das erste Prinzip auch als erster Demiurg angesehen wird, denn auch in diesem Text wird der noerische Helios Großer Demiurg genannt, was Julian wohl nicht gemacht hätte, wenn es einen noch viel Größeren gegeben hätte. Eine Möglichkeit wäre, dass die Göttermutter, die in der Heliosrede völlig abwesend war, hier aber oft als demiurgisch dargestellt wird, jetzt als erste Demiurgin betrachtet wird. Denn, so könnte man argumentieren, Helios' Titel als Großer Demiurg wäre wohl nicht so sehr bedroht, wenn seine Gattin die erste Demiurgin wäre, als wenn das erste Prinzip erster Demiurg wäre. Eine letzte Lösungsmöglichkeit bestünde in der These, dass mit dem Ausdruck ,dritter Demiurg' überhaupt nicht der sichtbare Helios gemeint ist. Dies könnte weiterhin als zweiter Demiurg - nach dem ersten Demiurg, dem noerischen Helios - betrachtet werden.

Wer könnte aber dieser dritte Demiurg sein, wenn es nicht Helios sein sollte ${ }^{183}$ Man könnte natürlich an Dionysos denken. Dagegen könnte aber sprechen, dass er, als partieller Demiurg, vielleicht nicht als Demiurg des gesamten fünften Körpers, einer universellen Substanz, betrachtet werden kann. Ein anderer Kandidat wäre Jamblichs ,Demiurg des Werdens' ( $\gamma \varepsilon v \varepsilon \sigma$ เovpyós), den der Philosoph aus Chalkis sowohl vom himmlischen als auch vom überhimmlischen, transzendenten Demiurgen und schließlich auch vom sublunaren Demiurgen zu unterscheiden scheint. ${ }^{184}$ Dieser, genesiourgische‘ Demiurg des Werdens könnte natürlich mit Dionysos identisch sein. Aber wenn Letzteres nicht der Fall ist und wir annehmen, dass Julian tatsächlich die Existenz eines ,genesiourgischen Demiurgen' annahm, gäbe es einen julianischen Demiurgen, den er in keinem seiner

102, 105) und Wright (S. 477, Anmerkung 1) behauptete Identifizierung von Attis mit dem Mondlicht (angeblich in 7,20-25 [167D]) ist eindeutig nicht vorhanden.

182 So sehen es beispielsweise Mau (Anmerkung 59), S. 93, und Bregman (Anmerkung 29), S. 343.

183 Es ist natürlich nicht so, dass nur Helios in Betracht kommt, weil nur er die Ursachen der Dinge in sich birgt. Dies könnte von jedem universellen, demiurgischen Gott gesagt werden. Selbstverständlich sind nicht alle Ursachen auf die gleiche Weise in jedem demiurgischen Gott vorhanden; dies ist genau der Grund, weshalb es mehrere Demiurgen geben muss.

184 Siehe Iamblichus, In Sophistem, Fragment 1, Zeile 14-16; 19 Dillon. 
philosophischen Werke erwähnt hat. ${ }^{185}$ Dies sollte aber keinen schwerwiegenden Einwand darstellen. Hätten wir nämlich die Rede auf die Göttermutter nicht gehabt, so hätten wir auch nie erfahren, dass Julian Attis als niedrigsten Demiurg und die Göttermutter als die Quelle der noerischen Götter betrachtete.

\section{Resümee}

Während Julian in der Streitschrift Gegen die Galiläer seine eigenen Auffassungen nur indirekt behandelt, stellen die Heliosrede und die Rede auf die Göttermutter positive Behandlungen ausgewählter, für die Demiurgie relevanter Themen dar. In keiner der beiden Reden wird aber ein vollständiges demiurgisches System auf methodisch begründende Weise dargestellt. Die Parallelen mit späteren Systemen, die uns besser erhalten sind, zeigen jedoch, dass Julians scheinbar divergierende Behauptungen in einem solchen System gründen, auch wenn er uns von diesem nur Bruchstücke bietet. Seine philosophische Hauptquelle ist dabei zweifelsohne Jamblich gewesen, dessen Ansichten, wie anzunehmen ist, auch die Basis für die Widerlegung jüdischer und christlicher Schöpfungs- und Gottesauffassungen bildeten.

185 In Contra Galileos, Fragment 19,36, wirft der Kaiser den Juden vor, dass ihr Gott keinen $\tau$ ô

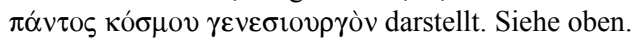

